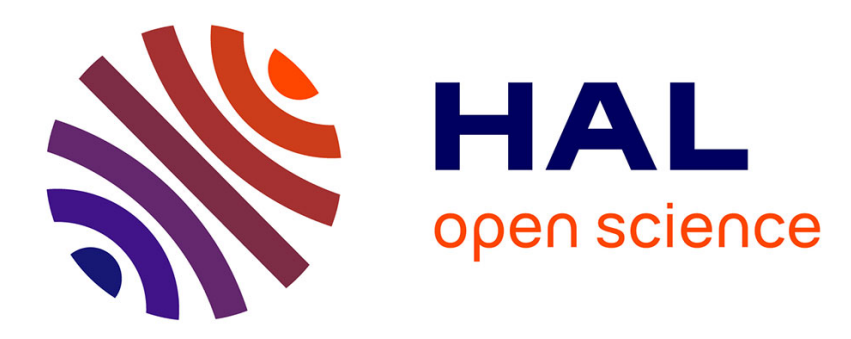

\title{
Numerical approximation of the general compressible Stokes problem
}

\author{
A Fettah, Thierry Gallouët
}

\section{To cite this version:}

A Fettah, Thierry Gallouët. Numerical approximation of the general compressible Stokes problem. IMA Journal of Numerical Analysis, 2013, 33 (3), pp.922 - 951. 10.1093/imanum/drs024 . hal01116755

\section{HAL Id: hal-01116755 \\ https://hal.science/hal-01116755}

Submitted on 14 Feb 2015

HAL is a multi-disciplinary open access archive for the deposit and dissemination of scientific research documents, whether they are published or not. The documents may come from teaching and research institutions in France or abroad, or from public or private research centers.
L'archive ouverte pluridisciplinaire HAL, est destinée au dépôt et à la diffusion de documents scientifiques de niveau recherche, publiés ou non, émanant des établissements d'enseignement et de recherche français ou étrangers, des laboratoires publics ou privés. 


\title{
NUMERICAL APPROXIMATION OF THE GENERAL COMPRESSIBLE STOKES PROBLEM
}

\author{
A. FETTAH AND T. GALLOUËT
}

\begin{abstract}
In this paper, we propose a discretization for the compressible Stokes problem with an equation of state of the form $p=\varphi(\rho)$ (where $p$ stands for the pressure, $\rho$ for the density and $\varphi$ is a superlinear nondecreasing function from $\mathbb{R}$ to $\mathbb{R}$ ). This scheme is based on Crouzeix-Raviart approximation spaces. The discretization of the momentum balance is obtained by the usual finite element technique. The discrete mass balance is obtained by a finite volume scheme, with an upwinding of the density, and two additional terms. We prove the existence of a discrete solution and the convergence of this approximate solution to a solution of the continuous problem.
\end{abstract}

\section{INTRODUCTION}

Let $\Omega$ be a bounded open set of $\mathbb{R}^{d}$, polygonal if $d=2$ and polyhedral if $d=3$. Let $\varphi \in C(\mathbb{R}, \mathbb{R})$ be a convex nondecreasing function such that:

$$
\varphi(0)=0, \varphi \text { is } C^{1} \text { on } \mathbb{R}_{+}^{\star}
$$

and

$$
\forall a \in \mathbb{R}, \exists b>0 \text { such that: } \varphi(s) \geq a s-b, \forall s \in \mathbb{R}_{+} .
$$

For $M, \mu>0, \boldsymbol{f} \in \mathrm{L}^{2}(\Omega)^{d}$ and $\boldsymbol{g} \in L^{\infty}(\Omega)^{d}$, we consider the following problem:

$$
\begin{aligned}
& -\mu \Delta \boldsymbol{u}-\frac{\mu}{3} \boldsymbol{\nabla}(\operatorname{div} \boldsymbol{u})+\nabla p=\boldsymbol{f}+\rho \boldsymbol{g} \text { in } \Omega, \boldsymbol{u}=0 \text { on } \partial \Omega, \\
& \operatorname{div}(\rho \boldsymbol{u})=0 \text { in } \Omega, \rho \geq 0 \text { in } \Omega, \int_{\Omega} \rho(x) \mathrm{d} \boldsymbol{x}=M, \\
& p=\varphi(\rho) \text { in } \Omega .
\end{aligned}
$$

Remark 1.1.

- We assume that the function $\varphi$ is convex, but not necessarily strictly convex. We also assume that $\varphi$ is nondecreasing but it can be constant on an interval (in fact, since $\varphi$ is convex, the function $\varphi$ is, at least for $m$ large enough, increasing on $[m,+\infty))$.

- The condition (1.1) is equivalent to the following one:

$$
\liminf _{s \rightarrow+\infty} \varphi(s) / s=+\infty
$$

Key words and phrases. Compressible Stokes equations, finite element methods, finite volume methods. 
- The fact that $\varphi(0)=0$ is not a restriction since $p$ can be replaced by $(p-\varphi(0))$ in the momemtum equation and the EOS (namely the equation $(1.2 \mathrm{c}))$ can be written as $p-\varphi(0)=\varphi(\rho)-\varphi(0)$.

- The convexity of the function $\varphi$ can be replaced by the following condition: there exist $a, \tilde{a}, b, \tilde{b}>0$ and $\gamma>1$ such that:

$$
\forall s \in \mathbb{R}_{+}, a s^{\gamma}-b \leq \varphi(s) \leq \tilde{a} s^{2 \gamma-1}+\tilde{b} .
$$

Here also the function $\varphi$ is assumed to be nondecreasing but not necessarily increasing.

- The coefficient $\mu / 3$ in the second term of the Left Hand Side of (1.2a) is natural from the physical point of view. From the mathematical point of view, it is easy to replace it by $\bar{\mu}$, as long as $\bar{\mu} \geq 0$.

Definition 1.2. Let $\boldsymbol{f} \in \mathrm{L}^{2}(\Omega)^{d}, \boldsymbol{g} \in L^{\infty}(\Omega)^{d}$ and $M>0$. A weak solution of Problem (1.2) is a function $(\boldsymbol{u}, p, \rho) \in \mathrm{H}_{0}^{1}(\Omega)^{d} \times \mathrm{L}^{2}(\Omega) \times \mathrm{L}^{2}(\Omega)$ satisfying:

$$
\begin{array}{r}
\mu \int_{\Omega} \nabla \boldsymbol{u}: \boldsymbol{\nabla} \boldsymbol{v} \mathrm{d} \boldsymbol{x}+\frac{\mu}{3} \int_{\Omega} \operatorname{div}(\boldsymbol{u}) \operatorname{div}(\boldsymbol{v}) \mathrm{d} \boldsymbol{x}-\int_{\Omega} p \operatorname{div}(\boldsymbol{v}) \mathrm{d} \boldsymbol{x} \\
=\int_{\Omega} \boldsymbol{f} \cdot \boldsymbol{v} \mathrm{d} \boldsymbol{x}+\int_{\Omega} \rho \boldsymbol{g} \cdot \boldsymbol{v} \mathrm{d} \boldsymbol{x} \text { for all } \boldsymbol{v} \in \mathrm{H}_{0}^{1}(\Omega)^{d}, \\
\quad \int_{\Omega} \rho \boldsymbol{u} \cdot \boldsymbol{\nabla} \varphi \mathrm{d} \boldsymbol{x}=0 \text { for all } \varphi \in \mathrm{W}^{1, \infty}(\Omega), \\
\rho \geq 0 \text { a.e. in } \Omega, \int_{\Omega} \rho \mathrm{d} \boldsymbol{x}=M, p=\varphi(\rho) \text { a.e. in } \Omega .
\end{array}
$$

The main objective of this paper is to present a numerical scheme for the computation of an approximate solution of Problem (1.2) and to prove the convergence (up to a subsequence, since, up to now, no uniqueness result is available for the solution of (1.2)) of this approximate solution towards a weak solution of (1.2) (i.e. a solution of (1.4)) as the mesh size goes to 0 . The present paper follows a previous paper [6] where a similar result was presented in the case $\varphi(\rho)=\rho^{\gamma}, \gamma>1$ (see also [11]). We present here a discretization with the so called Crouziex-Raviart element, as in [6]. However, it could be possible also, without additional difficulties, to use a MAC scheme, as in [7]. The fact to consider a general EOS (instead of $p=\rho^{\gamma}$ ) induces some additional difficulties with respect to the previous papers [6] and [7]. In particular for the estimates on the discrete solutions (Section 3.2 and Appendix A) and for passing to the limit in the EOS (Section 3.3 and Appendix B). For passing in the limit in the EOS, we mimic some ideas which were developped for the study of the Navier-Stokes equations, see [12], [8] or [13]. A part of the results given in this paper was presented in the FVCA6 workshop (Prague, 2011) and in a short paper (containing few proofs) in the proceedings of this workshop, see [9]. The present paper is more general. In particular, it considers more general EOS and it includes the gravity effects (two improvements which induce the need of non trivial developments, for instance for obtaining estimates on $u$ an $p$ and for passing to the limit in the EOS). Furthermore, the present paper contains complete proofs and an appendix with lemmas interesting for their own sake.

Remark 1.3. In the spirit of [12], [8] or [13] (which are devoted to the study of the compressible Navier-Stokes equations, but not on the discretization point of view), 
it is worth noticing that if $(\rho, u) \in L^{2}(\Omega) \times \mathrm{H}_{0}^{1}(\Omega)$ satisfies $(1.4 \mathrm{~b})$, then, it is known that $(\rho, u)$ is a renormalized solution of $\operatorname{div}(\rho u)=0$ in the sense of [4], that is

$$
\left(\rho \phi^{\prime}(\rho)-\phi(\rho)\right) \operatorname{div}(u)+\operatorname{div}(\phi(\rho) u)=0 \text { in } \mathcal{D}^{\prime}\left(\mathbb{R}^{d}\right),
$$

for any $C^{1}$-function $\phi$ from $\mathbb{R}$ to $\mathbb{R}$ such that $\phi^{\prime}$ is bounded (in order to give a sense to the preceding equation, we set $u=0$ in $\mathbb{R}^{d} \backslash \Omega$, so that $\left.u \in H^{1}\left(\mathbb{R}^{d}\right)\right)$. This is explained in Remark B.3.

\section{Discrete SPaCes AND Numerical SCheme}

Let $\mathcal{T}$ be a decomposition of the domain $\Omega$ in simplices, which we call herafter a triangulation of $\Omega$, regardless of the space dimension. By $\mathcal{E}(K)$, we denote the set of the edges $(d=2)$ or faces $(d=3) \sigma$ of the element $K \in \mathcal{T}$; for short, each edge or face will be called an edge hereafter. The set of all edges of the mesh is denoted by $\mathcal{E}$; the set of edges included in the boundary of $\Omega$ is denoted by $\mathcal{E}_{\text {ext }}$ and the set of internal edges (i.e. $\mathcal{E} \backslash \mathcal{E}_{\text {ext }}$ ) is denoted by $\mathcal{E}_{\text {int }}$. The decomposition $\mathcal{T}$ is assumed to be regular in the usual sense of the finite element literature (e.g. [2]), and, in particular, $\mathcal{T}$ satisfies the following properties: $\bar{\Omega}=\bigcup_{K \in \mathcal{T}} \bar{K}$; if $K, L \in \mathcal{T}$, then $\bar{K} \cap \bar{L}=\emptyset, \bar{K} \cap \bar{L}$ is a vertex or $\bar{K} \cap \bar{L}$ is a common edge of $K$ and $L$, which is denoted by $K \mid L$. For each internal edge of the mesh $\sigma=K \mid L, \boldsymbol{n}_{K L}$ stands for the normal vector of $\sigma$, oriented from $K$ to $L$ (so that $\boldsymbol{n}_{K L}=-\boldsymbol{n}_{L K}$ ). By $|K|$ and $|\sigma|$ we denote the ( $d$ and $d-1$ dimensional) measure, respectively, of an element $K$ and of an edge $\sigma$, and $h_{K}$ and $h_{\sigma}$ stand for the diameter of $K$ and $\sigma$, respectively. We measure the regularity of the mesh through the parameter $\theta$ defined by:

$$
\theta=\inf \left\{\frac{\xi_{K}}{h_{K}}, K \in \mathcal{T}\right\}
$$

where $\xi_{K}$ stands for the diameter of the largest ball included in $K$. Note that for all $\sigma \in \mathcal{E}_{\text {int }}, \sigma=K \mid L$, we have $h_{\sigma} \geq \xi_{K} \geq \theta h_{K}$ and $h_{\sigma} \leq h_{L}$ and so $\theta h_{K} \leq$ $h_{L} \leq \theta^{-1} h_{K}$. Note also that for all $K \in \mathcal{T}$ and for all $\sigma \in \mathcal{E}(K)$, the inequality $h_{\sigma}|\sigma| \leq 2 \theta^{-d}|K|$ holds ([10, relation (2.2)]) and if $\sigma=K \mid L$ a rough estimate gives $|K| \leq(2 / \theta)^{2 d}|L|$. These relations will be used throughout this paper. Finally, as usual, we denote by $h$ the quantity $\max _{K \in \mathcal{T}} h_{K}$.

The space discretization relies on the Crouzeix-Raviart element (see [3] for the seminal paper and, for instance, [5, pp. 199-201] for a synthetic presentation). The reference element is the unit $d$-simplex and the discrete functional space is the space $P_{1}$ of affine polynomials. The degrees of freedom are determined by the following set of edge functionals:

$$
\left\{F_{\sigma}, \sigma \in \mathcal{E}(K)\right\}, \quad F_{\sigma}(v)=|\sigma|^{-1} \int_{\sigma} v \mathrm{~d} \gamma
$$

The mapping from the reference element to the actual one is the standard affine mapping. Finally, the continuity of the average value of a discrete functions $v$ across each edge of the mesh, $F_{\sigma}(v)$, is required, thus the discrete space $V_{h}$ is defined as follows:

$$
\begin{aligned}
V_{h}=\left\{v \in L^{2}(\Omega): \forall K \in \mathcal{T},\left.v\right|_{K} \in P_{1}(K) ;\right. \\
\left.\quad \forall \sigma \in \mathcal{E}_{\text {int }}, \sigma=K \mid L, \quad F_{\sigma}\left(\left.v\right|_{K}\right)=F_{\sigma}\left(\left.v\right|_{L}\right) ; \forall \sigma \in \mathcal{E}_{\text {ext }}, F_{\sigma}(v)=0\right\} .
\end{aligned}
$$


Indeed, this space $V_{h}$ should be denoted by $V_{\mathcal{T}}$ since it depends on $\mathcal{T}$ and not only on $h$ (which is given by $\mathcal{T}$ ) but this (somewhat incorrect) notation is commonly used.

The space of approximation for the velocity is the space $\boldsymbol{W}_{h}$ of vector-valued functions each component of which belongs to $V_{h}: \boldsymbol{W}_{h}=\left(V_{h}\right)^{d}$. The pressure and the density are approximated by the space $L_{h}$ of piecewise constant functions:

$$
L_{h}=\left\{q \in L^{2}(\Omega):\left.q\right|_{K}=\text { constant, } \forall K \in \mathcal{T}\right\} .
$$

Since only the continuity of the integral over each edge of the mesh is imposed, the functions of $V_{h}$ are discontinuous through each edge; the discretization is thus nonconforming in $H^{1}(\Omega)^{d}$. We then define, for $1 \leq i \leq d$ and $\boldsymbol{u} \in V_{h}, \partial_{h, i} \boldsymbol{u}$ as the function of $\mathrm{L}^{2}(\Omega)$ which is equal to the derivative of $u$ with respect to the $i^{\text {th }}$ space variable almost everywhere. This notation allows to define the discrete gradient, denoted by $\nabla_{h}$, for both scalar and vector-valued discrete functions and the discrete divergence of vector-valued discrete functions, denoted by $\operatorname{div}_{h}$.

The Crouzeix-Raviart pair of approximation spaces for the velocity and the pressure is inf-sup stable, in the usual sense for "piecewise $\mathrm{H}^{1}$ " discrete velocities, i.e. there exists $c_{\mathrm{i}}>0$ only depending on $\Omega$ and, in a non-increasing way, on $\theta$, such that:

$$
\forall p \in L_{h}, \quad \sup _{\boldsymbol{v} \in \boldsymbol{W}_{h}} \frac{\int_{\Omega} p \operatorname{div}_{h}(\boldsymbol{v}) \mathrm{d} \boldsymbol{x}}{\|\boldsymbol{v}\|_{1, b}} \geq c_{\mathrm{i}}\|p-m(p)\|_{\mathrm{L}^{2}(\Omega)},
$$

where $m(p)$ is the value of $p$ over $\Omega$ and $\|\cdot\|_{1, b}$ stands for the broken Sobolev $\mathrm{H}^{1}$ semi-norm, which is defined for scalar as well as for vector-valued functions by:

$$
\|v\|_{1, b}^{2}=\sum_{K \in \mathcal{T}} \int_{K}|\nabla v|^{2} \mathrm{~d} \boldsymbol{x}=\int_{\Omega}\left|\nabla_{h} v\right|^{2} \mathrm{~d} \boldsymbol{x} .
$$

This norm is known to control the $\mathrm{L}^{2}$ norm by a Poincaré inequality (e.g. [5, lemma 3.31]). We also define a discrete semi-norm on $L_{h}$, similar to the usual $\mathrm{H}^{1}$ semi-norm used in the finite volume context:

$$
\forall \rho \in L_{h}, \quad|\rho|_{\mathcal{T}}^{2}=\sum_{\substack{\sigma \in \mathcal{E}_{\text {int }}, \sigma=K \mid L}} \frac{|\sigma|}{h_{\sigma}}\left(\rho_{K}-\rho_{L}\right)^{2} .
$$

From the definition (2.2), each velocity degree of freedom may be indexed by the number of the component and the associated edge, thus the set of velocity degrees of freedom reads:

$$
\left\{v_{\sigma, i}, \sigma \in \mathcal{E}_{\mathrm{int}}, 1 \leq i \leq d\right\} .
$$

We denote by $e_{\sigma}$ the usual Crouzeix-Raviart shape function associated to $\sigma$, i.e. the scalar function of $V_{h}$ such that $F_{\sigma}\left(e_{\sigma}\right)=1$ and $F_{\sigma^{\prime}}\left(e_{\sigma}\right)=0$, for all $\sigma^{\prime} \in \mathcal{E} \backslash\{\sigma\}$.

Similarly, each degree of freedom for the pressure is associated to a cell $K$, and the set of pressure degrees of freedom is denoted by $\left\{p_{K}, K \in \mathcal{T}\right\}$.

We define by $r_{h}$ the following interpolation operator:

$$
r_{h}: \quad \begin{array}{lll}
\mathrm{H}_{0}^{1}(\Omega) & \longrightarrow & V_{h} \\
u & \mapsto & r_{h} u=\sum_{\sigma \in \mathcal{E}} F_{\sigma}(u) e_{\sigma}=\sum_{\sigma \in \mathcal{E}}|\sigma|^{-1}\left(\int_{\sigma} v \mathrm{~d} \gamma\right) e_{\sigma} .
\end{array}
$$


This operator naturally extends to vector-valued functions (i.e. to perform the interpolation from $\mathrm{H}_{0}^{1}(\Omega)^{d}$ to $\left.\boldsymbol{W}_{h}\right)$ and we keep the same notation $r_{h}$ for both the scalar and vector case. The properties of $r_{h}$ are gathered in the following lemma. They are proven in [3].

Theorem 2.1. Let $\theta_{0}>0$ and let $\mathcal{T}$ be a triangulation of the computational domain $\Omega$ such that $\theta \geq \theta_{0}$, where $\theta$ is defined by (2.1). The interpolation operator $r_{h}$ enjoys the following properties:

(1) preservation of the divergence:

$$
\forall \boldsymbol{v} \in \mathrm{H}_{0}^{1}(\Omega)^{d}, \forall q \in L_{h}, \quad \int_{\Omega} q \operatorname{div}_{h}\left(r_{h} \boldsymbol{v}\right) \mathrm{d} \boldsymbol{x}=\int_{\Omega} q \operatorname{div}(\boldsymbol{v}) \mathrm{d} \boldsymbol{x},
$$

(2) stability:

$$
\forall v \in \mathrm{H}_{0}^{1}(\Omega), \quad\left\|r_{h} v\right\|_{1, b} \leq c_{1}\left(\theta_{0}\right)|v|_{\mathrm{H}^{1}(\Omega)},
$$

(3) approximation properties:

$$
\begin{aligned}
& \forall v \in \mathrm{H}^{2}(\Omega) \cap \mathrm{H}_{0}^{1}(\Omega), \forall K \in \mathcal{T}, \\
& \quad\left\|v-r_{h} v\right\|_{\mathrm{L}^{2}(K)}+h_{K}\left\|\nabla_{h}\left(v-r_{h} v\right)\right\|_{\mathrm{L}^{2}(K)} \leq c_{2}\left(\theta_{0}\right) h_{K}^{2}|v|_{\mathrm{H}^{2}(K)} .
\end{aligned}
$$

In both above inequalities, the notation $c_{i}\left(\theta_{0}\right)$ means that the real number $c_{i}$ only depends on $\theta_{0}$ and $\Omega$, and, in particular, does not depend on the parameter $h$ characterizing the size of the cells; this notation will be kept throughout the paper.

The following compactness result was proven in [10, Theorem 3.3].

Theorem 2.2. Let $\left(v_{n}\right)_{n \in \mathbb{N}}$ be a sequence of functions satisfying the following assumptions:

(1) $\forall n \in \mathbb{N}$, there exists a triangulation of the domain $\mathcal{T}_{n}$ such that $v_{n} \in V_{h_{n}}$, where $V_{h_{n}}$ is the space of Crouzeix-Raviart discrete functions associated to $\mathcal{T}_{n}$ (and $h_{n}$ given by $\mathcal{T}_{n}$ ), as defined by $(2.3)$, and the parameter $\theta_{n}$ characterizing the regularity of $\mathcal{T}_{n}$ is bounded away from zero independently of $n$,

(2) the sequence $\left(v_{n}\right)_{n \in \mathbb{N}}$ is uniformly bounded with respect to the broken Sobolev $\mathrm{H}^{1}$ semi-norm, i.e.:

$$
\forall n \in \mathbb{N}, \quad\left\|v_{n}\right\|_{1, b} \leq C,
$$

where $C$ is a constant real number and $\|\cdot\|_{1, b}$ stands for the broken Sobolev $\mathrm{H}^{1}$ semi-norm associated to $\mathcal{T}_{n}$ (with a slight abuse of notation, namely dropping, for short, the index $n$ pointing the dependence of the norm with respect to the mesh).

Then, when $n \rightarrow \infty$, possibly up to the extraction of a subsequence, the sequence $\left(v_{n}\right)_{n \in \mathbb{N}}$ converges (strongly) in $\mathrm{L}^{2}(\Omega)$ to a limit $\bar{v}$ such that $\bar{v} \in \mathrm{H}_{0}^{1}(\Omega)$.

We now present the numerical scheme we use. Let $\rho^{*}$ be the mean density, i.e. $\rho^{*}=M /|\Omega|$ where $|\Omega|$ stands for the measure of the domain $\Omega$. Let also $\alpha$ and $\xi$ be given, with $\alpha>0$ and $0<\xi<2$. Let $\mathcal{T}$ be a (regular) decomposition of the domain $\Omega$ in simplices. The discrete unknowns are $\boldsymbol{u}, p$ and $\rho$, with $\boldsymbol{u} \in \boldsymbol{W}_{h}$ and $p, \rho \in L_{h}$. Using the notations previously introduced, we consider the following numerical scheme for the discretization of Problem (1.2): 


$$
\begin{array}{r}
\mu \int_{\Omega} \nabla_{h} \boldsymbol{u}: \nabla_{h} \boldsymbol{v} \mathrm{d} \boldsymbol{x}+\frac{\mu}{3} \int_{\Omega} \operatorname{div}_{h}(\boldsymbol{u}) \operatorname{div}_{h}(\boldsymbol{v}) \mathrm{d} \boldsymbol{x}-\int_{\Omega} p \operatorname{div}_{h}(\boldsymbol{v}) \mathrm{d} \boldsymbol{x} \\
=\int_{\Omega} \boldsymbol{f} \cdot \boldsymbol{v} \mathrm{d} \boldsymbol{x}+\int_{\Omega} \rho \boldsymbol{g} \cdot \boldsymbol{v} \mathrm{d} \boldsymbol{x} \text { for all } \boldsymbol{v} \in \boldsymbol{W}_{h}, \\
\sum_{\sigma=K \mid L}\left(|\sigma| \boldsymbol{u}_{K, \sigma}^{+} \rho_{K}-|\sigma| \boldsymbol{u}_{K, \sigma}^{-} \rho_{L}\right)+M_{K}+T_{K}=0 \text { for all } K \in \mathcal{T}, \\
p_{K}=\varphi\left(\rho_{K}\right) \text { for all } K \in \mathcal{T} .
\end{array}
$$

The quantity $\boldsymbol{u}_{K, \sigma}$ is defined by

$$
\boldsymbol{u}_{K, \sigma}=|\sigma|^{-1} \int_{\sigma} \boldsymbol{u} \mathrm{d} \gamma \cdot \boldsymbol{n}_{K L} .
$$

As usual, $\boldsymbol{u}_{K, \sigma}^{+}=\max \left(\boldsymbol{u}_{K, \sigma}, 0\right)$ and $\boldsymbol{u}_{K, \sigma}^{-}=-\min \left(\boldsymbol{u}_{K, \sigma}, 0\right)$, so that $\boldsymbol{u}_{K, \sigma}=\boldsymbol{u}_{K, \sigma}^{+}-$ $\boldsymbol{u}_{K, \sigma}^{-}$. The terms $M_{K}$ and $T_{K}$ read:

$$
\begin{aligned}
& M_{K}=h^{\alpha}|K|\left(\rho_{K}-\rho^{*}\right), \\
& T_{K}=\sum_{\sigma=K \mid L} h^{\xi} \frac{|\sigma|}{h_{\sigma}}\left(\left|\rho_{K}\right|+\left|\rho_{L}\right|\right)\left(\rho_{K}-\rho_{L}\right) .
\end{aligned}
$$

\section{Existence AND CONVERGENCE OF APPROXimate SOlutions}

3.1. Existence of a solution. Let $\mathcal{T}$ be a (regular) decomposition of the domain $\Omega$ in simplices. We prove in this section the existence of a discrete solution, that the existence of a solution to (2.5), by using the Brouwer fixed point theorem to a convenient application $T$ from $\mathbb{R}^{N}$ to $\mathbb{R}^{N}$ where $N=\operatorname{card}(\mathcal{T})$. We first define $T$.

Let $\tilde{\rho}=\left(\tilde{\rho}_{K}\right)_{K \in \mathcal{T}}$. Choosing the elements of $\mathcal{T}$ in an arbitrary order, we then have $\tilde{\rho} \in \mathbb{R}^{N}$. We calculate $p$ by the following relation: $p_{K}=\varphi\left(\tilde{\rho}_{K}^{+}\right)$for all $K \in \mathcal{T}$.

We now compute $\boldsymbol{u}$ as the unique solution (in $\boldsymbol{W}_{h}$ ) of (2.5a) with $\tilde{\rho}$ instead of $\rho$ in the Right Hand Side of $(2.5 \mathrm{a})$ (and $p$ given by $p_{K}=\varphi\left(\tilde{\rho}_{K}^{+}\right)$for all $K \in \mathcal{T}$ ). The existence and uniqueness of $\boldsymbol{u}$ is an easy consequence of the coercivity in $\boldsymbol{W}_{h}$ of the bilinear form

$$
(u, v) \mapsto \mu \int_{\Omega} \nabla_{h} \boldsymbol{u}: \nabla_{h} \boldsymbol{v} \mathrm{d} \boldsymbol{x} .
$$

Furthermore, the solution $\boldsymbol{u}$ continuously depends on $\tilde{\rho}$ (since $\varphi$ is continuous).

We have now to define $\rho$ (and we will set $T(\tilde{\rho})=\rho$ ). We change a little bit the term $T_{K}$. Instead of $(2.6 \mathrm{~b})$, we take

$$
T_{K}=\sum_{\sigma=K \mid L} h^{\xi} \frac{|\sigma|}{h_{\sigma}}\left(\left|\tilde{\rho}_{K}\right|+\left|\tilde{\rho}_{L}\right|\right)\left(\rho_{K}-\rho_{L}\right) .
$$

With this choice of $T_{K}$, the set of Equations (2.5b) leads to the linear system of $N$ equations with $N$ unknowns (which are $\rho_{K}$ for $K \in \mathcal{T}$ ). The equations of this system may be written as:

$$
\sum_{L \in \mathcal{T}} a_{K, L} \rho_{L}=b_{K} \text { for all } K \in \mathcal{T}
$$


with

$$
\begin{gathered}
a_{K, K}=h^{\alpha}|K|+\sum_{\sigma=K \mid L}\left(|\sigma| u_{K, \sigma}^{+}+h^{\xi} \frac{|\sigma|}{h_{\sigma}}\left(\left|\tilde{\rho}_{K}\right|+\left|\tilde{\rho}_{L}\right|\right)\right), \\
a_{K, L}=-|\sigma| u_{K, \sigma}^{-}-h^{\xi} \frac{|\sigma|}{h_{\sigma}}\left(\left|\tilde{\rho}_{K}\right|+\left|\tilde{\rho}_{L}\right|\right) \text { if } \sigma=K \mid L \\
a_{K, L}=0 \text { if } K \text { and } L \text { do not share an interface. }
\end{gathered}
$$

$$
b_{K}=h^{\alpha}|K| \rho^{\star} .
$$

Using the fact that $u_{L, \sigma}^{-}=u_{K, \sigma}^{+}($for $\sigma=K \mid L)$, one has, for all $K \in \mathcal{T}$,

$$
\sum_{L \in \mathcal{T}} a_{K, L}>0
$$

and, for all $K, L \in \mathcal{T}, K \neq L$,

$$
a_{K, L} \leq 0 .
$$

With these properties, it is quite easy to show thet the system (3.1) has a unique solution. Furthermore, since $b_{K}>0$ for all $K \in \mathcal{T}$ the solution $\rho$ satisfy $\rho_{K}>0$ for all $K \in \mathcal{T}$ (see Lemma C.4). Finally, since the coeffcients $a_{K, L}$ and $b_{K}$ depend continuously of $\tilde{\rho}$ (and since the application $A \mapsto A^{-1}$ is continuous on the set of invertible $N \times N$ matrix), the solution $\rho$ of (3.1) continuously depends on $\tilde{\rho}$.

We define now (as we said before) the map $T$ from $\mathbb{R}^{N}$ to $\mathbb{R}^{N}$ setting $T(\tilde{\rho})=\rho$. The map $T$ is continuous.

If $\rho \in \operatorname{Im}(T)$, we also showed that $\rho_{K}>0$ for all $K \in \mathcal{T}$. Futhermore summing for $K \in \mathcal{T}$ the equations (3.1) we obtain

$$
\left.\sum_{K \in \mathcal{T}} h^{\alpha} \mid K\right] \rho_{K}=\sum_{K \in \mathcal{T}} b_{K}=\sum_{K \in \mathcal{T}} h^{\alpha}|K| \rho^{\star} .
$$

With the definition of $\rho^{\star}$, this gives $\sum_{K \in \mathcal{T}}|K| \rho_{K}=M$. Since $\rho \mapsto \sum_{K \in \mathcal{T}}|K|\left|\rho_{K}\right|$ is a norm on $\mathbb{R}^{N}$, this proves that the whole set $\operatorname{Im}(T)$ is included in a fixed ball of $\mathbb{R}^{N}$. Then, we can apply the Brouwer fixed point theorem. It gives the existence of $\rho \in \mathbb{R}^{N}$ such that $T(\rho)=\rho$. This gives the existence of a solution $(\boldsymbol{u}, p, \rho)$ to $(2.5)$.

We conclude this section by remarking that if $(\boldsymbol{u}, p, \rho)$ is a solution to (2.5), we necessarily have $T(\rho)=\rho$ and this show that

$$
\rho_{K}>0 \text { for all } K \in \mathcal{T} \text { and } \sum_{K \in \mathcal{T}}|K| \rho_{K}=M \text {. }
$$

\subsection{Estimates on the discrete solution.}

Lemma 3.1. Let $\mathcal{T}$ be a triangulation of the computational domain $\Omega$ and $\Phi$ a nondecreasing function in $C^{1}\left(\mathbb{R}_{*}^{+}\right)$. Let $(\boldsymbol{u}, \rho) \in \boldsymbol{W}_{h} \times L_{h}$ satisfy the second equation of the scheme, i.e. Equation (2.5b). Then, $\rho_{K}>0$ for all $K \in \mathcal{T}$ and:

$$
\int_{\Omega} \Phi(\rho) \operatorname{div}_{h}(\boldsymbol{u}) \mathrm{d} \boldsymbol{x} \leq 0 .
$$


Proof. We fist remark that $\rho$ is solution of (3.1) with

$$
\begin{gathered}
a_{K, K}=h^{\alpha}|K|+\sum_{\sigma=K \mid L}\left(|\sigma| u_{K, \sigma}^{+}+h^{\xi} \frac{|\sigma|}{h_{\sigma}}\left(\left|\rho_{K}\right|+\left|\rho_{L}\right|\right)\right), \\
a_{K, L}=-|\sigma| u_{K, \sigma}^{-}-h^{\xi} \frac{|\sigma|}{h_{\sigma}}\left(\left|\rho_{K}\right|+\left|\rho_{L}\right|\right) \text { if } \sigma=K \mid L, \\
a_{K, L}=0 \text { if } K \text { and } L \text { do not share an interface. } \\
b_{K}=h^{\alpha}|K| \rho^{\star} .
\end{gathered}
$$

Then, since $b_{K}>0$ for all $K \in \mathcal{T}$, one has $\rho_{K}>0$ for all $K \in \mathcal{T}$ (see Lemma C.4).

Let the function $\psi \in C^{1}\left(\mathbb{R}_{+}^{\star}\right)$ satisfying $\psi^{\prime}(s)=\frac{\Phi^{\prime}(s)}{s}$ for all $s>0(\psi$ is nondecreasing). Multiplying (2.5b) by $\psi\left(\rho_{K}\right)$ and summing over $K \in \mathcal{T}$ yields $T_{1}+T_{2}+T_{3}=0$ with:

$$
\begin{aligned}
T_{1} & =\sum_{K \in \mathcal{T}} \psi\left(\rho_{K}\right) \sum_{\sigma=K \mid L}|\sigma| \rho_{\sigma} \boldsymbol{u}_{\sigma} \cdot \boldsymbol{n}_{K L}, \\
T_{2} & =\sum_{K \in \mathcal{T}} h^{\alpha}|K| \psi\left(\rho_{K}\right)\left(\rho_{K}-\rho^{*}\right), \\
T_{3} & =\sum_{K \in \mathcal{T}} \psi\left(\rho_{K}\right) \sum_{\sigma=K \mid L}\left(h_{K}+h_{L}\right)^{\xi} \frac{|\sigma|}{h_{\sigma}}\left(\rho_{K}+\rho_{L}\right)\left(\rho_{K}-\rho_{L}\right) .
\end{aligned}
$$

Let:

$$
T_{4}=\sum_{K \in \mathcal{T}} \int_{K} \Phi\left(\rho_{K}\right) \operatorname{div}(\boldsymbol{u})=\sum_{\sigma=K \mid L}|\sigma| \boldsymbol{u}_{\sigma} \cdot \boldsymbol{n}_{K L}\left(\Phi\left(\rho_{K}\right)-\Phi\left(\rho_{L}\right)\right)
$$

We have: $T_{4}=T_{4}-T_{1}-T_{2}-T_{3}$

$$
=\sum_{\sigma=K \mid L}|\sigma| \boldsymbol{u}_{\sigma} \cdot \boldsymbol{n}_{K L}\left[\Phi\left(\rho_{K}\right)-\Phi\left(\rho_{L}\right)-\rho_{\sigma}\left(\psi\left(\rho_{K}\right)-\psi\left(\rho_{L}\right)\right)\right]-T_{2}-T_{3},
$$

with $\rho_{\sigma}=\rho_{K}$ if $\boldsymbol{u}_{\sigma} \cdot \boldsymbol{n}_{K L}>0$ and $\rho_{\sigma}=\rho_{L}$ if $\boldsymbol{u}_{\sigma} \cdot \boldsymbol{n}_{K L}<0$.

The fact that $\psi$ is nondecreasing yields:

$\star T_{2} \geq \sum_{K \in \mathcal{T}} h^{\alpha}|K| \psi\left(\rho^{*}\right)\left(\rho_{K}-\rho^{*}\right)=0$,

$\star T_{3}=\sum_{\sigma=K \mid L}\left(h_{K}+h_{L}\right)^{\xi} \frac{|\sigma|}{h_{\sigma}}\left(\rho_{K}+\rho_{L}\right)\left(\rho_{K}-\rho_{L}\right)\left(\psi\left(\rho_{K}\right)-\psi\left(\rho_{L}\right)\right) \geq 0$.

For $\alpha>0$, we define $\Phi_{\alpha}$ on $\mathbb{R}_{+}^{\star}$ by $\Phi_{\alpha}(s)=\Phi(\alpha)-\Phi(s)-\alpha(\psi(\alpha)-\psi(s))$. Since $\Phi$ is nondecreasing (and $s \psi^{\prime}(s)=\Phi^{\prime}(s)$ ), one has $\Phi_{\alpha}(s) \leq 0$ for all $s \in \mathbb{R}_{+}^{*}$. Then, thanks to the choice of $\rho_{\sigma}$, one has

$$
\sum_{\sigma=K \mid L}|\sigma| \boldsymbol{u}_{\sigma} \cdot \boldsymbol{n}_{K L}\left[\Phi\left(\rho_{K}\right)-\Phi\left(\rho_{L}\right)-\rho_{\sigma}\left(\psi\left(\rho_{K}\right)-\psi\left(\rho_{L}\right)\right)\right] \leq 0
$$

which gives:

$$
T_{4}=\int_{\Omega} \Phi(\rho) \operatorname{div}_{h}(\boldsymbol{u}) \mathrm{d} \boldsymbol{x} \leq 0
$$

Proposition 3.2. Let $\theta_{0}>0$ and let $\mathcal{T}$ be a triangulation of the computational domain $\Omega$ such that $\theta \geq \theta_{0}$, where $\theta$ is defined by (2.1). Let $(\boldsymbol{u}, p, \rho) \in \boldsymbol{W}_{h} \times L_{h} \times L_{h}$ be a solution of (2.5). Then there exists $C$, only depending on the data of the problem $\Omega, \boldsymbol{f}, \boldsymbol{g}, \mu, \varphi, M$ and on $\theta_{0}$, such that: 


$$
\|\boldsymbol{u}\|_{1, b} \leq C,\|p\|_{\mathrm{L}^{2}(\Omega)} \leq C \text { and }\|\rho\|_{\mathrm{L}^{2}(\Omega)} \leq C .
$$

Proof. Let $(\boldsymbol{u}, p, \rho)$ be a solution of (2.5) . Taking $\boldsymbol{u}$ as test function in (2.5a) yields:

$$
\mu\|\boldsymbol{u}\|_{1, b}^{2}+\frac{\mu}{3} \int_{\Omega} \operatorname{div}_{h}^{2}(\boldsymbol{u}) \mathrm{d} \boldsymbol{x}-\int_{\Omega} p \operatorname{div}_{h}(\boldsymbol{u}) \mathrm{d} \boldsymbol{x}=\int_{\Omega} \boldsymbol{f} \cdot \boldsymbol{u} \mathrm{d} \boldsymbol{x}+\int_{\Omega} \rho \boldsymbol{g} \cdot \boldsymbol{u} \mathrm{d} \boldsymbol{x} .
$$

Using Lemma 3.1, a (well known) discrete Poincaré Inequality and the Hölder Inequality, one obtains the existence of $C_{1}$ only depending on $\Omega, \boldsymbol{f}, \mu, \boldsymbol{g}$ such that

$$
\|\boldsymbol{u}\|_{1, b} \leq C_{1}\left(1+\|\rho\|_{\mathrm{L}^{2}(\Omega)}\right) .
$$

Since $p=\varphi(\rho)$, using (1.1), for all $\varepsilon>0$ there exists $C_{\varepsilon}$ (only depending on $\varepsilon, \varphi$ and $\Omega$ ) such that:

$$
\|\rho\|_{\mathrm{L}^{2}(\Omega)} \leq C_{\varepsilon}+\varepsilon\|p\|_{\mathrm{L}^{2}(\Omega)} .
$$

Then, with (3.4), for all $\varepsilon>0$, there exists $\bar{C}_{\varepsilon}$, only depending on $\Omega, \boldsymbol{f}, \mu, \boldsymbol{g}, \varphi$ and $\varepsilon$ such that

$$
\|\boldsymbol{u}\|_{1, b} \leq \bar{C}_{\varepsilon}+\varepsilon\|p\|_{\mathrm{L}^{2}(\Omega)} .
$$

We now use Lemma C.2. There exists $\boldsymbol{w} \in \mathrm{H}_{0}^{1}(\Omega)^{d}$ such that $\operatorname{div}(\boldsymbol{w})=p-m(p)$ a.e. in $\Omega$ and $\|\boldsymbol{w}\|_{\mathrm{H}^{1}(\Omega)^{d}} \leq c_{2}\|p-m(p)\|_{\mathrm{L}^{2}(\Omega)}$ where $c_{2}$ only depends on $\Omega$.

Taking $\boldsymbol{v}=r_{h} \boldsymbol{w}$ as test function in (2.5a) yields:

$$
\begin{array}{r}
\int_{\Omega} p \operatorname{div}_{h}(\boldsymbol{v}) \mathrm{d} \boldsymbol{x}=\mu \int_{\Omega} \boldsymbol{\nabla}_{h} \boldsymbol{u}: \boldsymbol{\nabla}_{h} \boldsymbol{v} \mathrm{d} \boldsymbol{x}+\frac{\mu}{3} \int_{\Omega} \operatorname{div}_{h}(\boldsymbol{u}) \operatorname{div}_{h}(\boldsymbol{v}) \mathrm{d} \boldsymbol{x} \\
-\int_{\Omega} \boldsymbol{f} \cdot \boldsymbol{v} \mathrm{d} \boldsymbol{x}-\int_{\Omega} \rho \boldsymbol{g} \cdot \boldsymbol{v} \mathrm{d} \boldsymbol{x} .
\end{array}
$$

Since $\int_{\Omega} \operatorname{div}_{h}(\boldsymbol{v}) \mathrm{d} \boldsymbol{x}=0$, this gives also

$$
\begin{array}{r}
\int_{\Omega}[p-m(p)] \operatorname{div}_{h}(\boldsymbol{v}) \mathrm{d} \boldsymbol{x}=\mu \int_{\Omega} \boldsymbol{\nabla}_{h} \boldsymbol{u}: \boldsymbol{\nabla}_{h} \boldsymbol{v} \mathrm{d} \boldsymbol{x}+\frac{\mu}{3} \int_{\Omega} \operatorname{div}_{h}(\boldsymbol{u}) \operatorname{div}_{h}(\boldsymbol{v}) \mathrm{d} \boldsymbol{x} \\
-\int_{\Omega} \boldsymbol{f} \cdot \boldsymbol{v} \mathrm{d} \boldsymbol{x}-\int_{\Omega} \rho \boldsymbol{g} \cdot \boldsymbol{v} \mathrm{d} \boldsymbol{x}
\end{array}
$$

and then

$$
\begin{array}{r}
\int_{\Omega}[p-m(p)]^{2} \mathrm{~d} \boldsymbol{x}=\mu \int_{\Omega} \boldsymbol{\nabla}_{h} \boldsymbol{u}: \boldsymbol{\nabla}_{h} \boldsymbol{v} \mathrm{d} \boldsymbol{x}+\frac{\mu}{3} \int_{\Omega} \operatorname{div}_{h}(\boldsymbol{u}) \operatorname{div}_{h}(\boldsymbol{v}) \mathrm{d} \boldsymbol{x} \\
-\int_{\Omega} \boldsymbol{f} \cdot \boldsymbol{v} \mathrm{d} \boldsymbol{x}-\int_{\Omega} \rho \boldsymbol{g} \cdot \boldsymbol{v} \mathrm{d} \boldsymbol{x} .
\end{array}
$$

Using theorem 2.1, lemma C.2 and the inequalities (3.5) and (3.6) we get for all $\varepsilon>0$, the existence of $D_{\varepsilon}$, only depending on $\Omega, \boldsymbol{f}, \mu, \boldsymbol{g}, \varphi, \theta_{0}$ and $\varepsilon$ such that

$$
\|p-m(p)\|_{\mathrm{L}^{2}(\Omega)} \leq D_{\varepsilon}+\varepsilon\|p\|_{\mathrm{L}^{2}(\Omega)} .
$$

In order to obtain an estimate on $\|p\|_{L^{2}}$, we now use the fact that $\int_{\Omega} \rho d x=M$ (and we will deduce an estimate on $\|p\|_{L^{2}}$ in term of $\Omega, \boldsymbol{f}, \mu, \boldsymbol{g}, \theta_{0}, \varphi$ and $M$ ).

We first modify a little bit the function $\varphi$ (which is only nondecreasing) in order to obtain a function $\bar{\varphi}$ continuous and one-to-one from $\mathbb{R}_{+}$onto $\mathbb{R}_{+}$, so as to be 
able to use its inverse function. Let $s_{0}>0$ such that $\varphi\left(s_{0}\right)=1$. We define the increasing function $\bar{\varphi}$ from $\mathbb{R}_{+}$to $\mathbb{R}_{+}$by

$$
\begin{aligned}
& \bar{\varphi}(s)=\frac{s}{s_{0}} \text { if } 0 \leq s \leq s_{0}, \\
& \bar{\varphi}(s)=s \max _{s \in\left[s_{0}, s\right]} \frac{\varphi(t)}{t} \text { if } s_{0}<s .
\end{aligned}
$$

The function $\bar{\varphi}$ is a continuous increasing and one-to-one function from $\mathbb{R}_{+}$onto $\mathbb{R}_{+}$. Then, there exists $\psi$ (continuous increasing and one-to-one) from $\mathbb{R}_{+}$onto $\mathbb{R}_{+}$ such that

$$
\psi(\bar{\varphi}(s))=\bar{\varphi}(\psi(s))=s \text { for all } s \in \mathbb{R}_{+} .
$$

Since $\operatorname{Im}(\psi)=\mathbb{R}_{+}$, we have $\lim _{s \rightarrow+\infty} \psi(s)=+\infty$.

We also remark that for all $s \geq 0$ one has for $s \geq s_{0}, \bar{\varphi}(s) \geq \varphi(s)$ and then, a.e. in $\Omega$,

$$
\psi(p)=\psi(\varphi(\rho)) \leq \psi(\bar{\varphi}(\rho))+\varphi\left(s_{0}\right)=\rho+1 .
$$

This gives $\int_{\Omega} \psi(p) d x \leq M+\lambda_{d}(\Omega)$.

We now use Lemma A.1. It gives the existence of $\bar{C}$, only depending on $\Omega, \boldsymbol{f}, \mu$, $\boldsymbol{g}, \theta_{0}, \varphi$ and $M$ such that

$$
\|p\|_{L^{2}} \leq \bar{C} .
$$

Using (3.7) in (3.4) we thus get the estimate on $\|\boldsymbol{u}\|_{1, b}$.

Finally, thanks to $p=\varphi(\rho)$ and (1.1), the estimate on $\rho$ follows.

Lemma 3.3. Let $\theta_{0}>0$ and let $\mathcal{T}$ be a triangulation of the computational domain $\Omega$ such that $\theta \geq \theta_{0}$, where $\theta$ is defined by (2.1). Let $(\boldsymbol{u}, p, \rho) \in \boldsymbol{W}_{h} \times L_{h} \times L_{h}$ be a solution of (2.5). Then, there exists $\bar{C}$ only depending on $\Omega, \boldsymbol{f}, \boldsymbol{g}, \mu, \varphi, M$ and $\theta_{0}$ such that

$$
\begin{array}{r}
h^{\xi}|\rho|_{\mathcal{T}}^{2} \leq \bar{C} \text { and } E(\rho) \leq \bar{C} \\
\text { where } E(\rho)=\sum_{\sigma=K \mid L} \min \left(\frac{1}{\rho_{K}}, \frac{1}{\rho_{L}}\right)\left(\rho_{K}-\rho_{L}\right)^{2}|\sigma|\left|\boldsymbol{u}_{K, \sigma}\right|
\end{array}
$$

Proof. We recall that $\rho_{K}>0$ for all $K \in \mathcal{T}$. Multiplying Equation (2.5b) by $\ln (\rho(K))$ and summing over $K \in \mathcal{T}$, we thus obtain:

$$
\sum_{K \in \mathcal{T}} \ln \left(\rho_{K}\right) \sum_{\sigma=K \mid L}|\sigma| \boldsymbol{u}_{K, \sigma} \rho_{\sigma}+\sum_{K \in \mathcal{T}} \ln \left(\rho_{K}\right) M_{K}+\sum_{K \in \mathcal{T}} \ln \left(\rho_{K}\right) T_{K}=0,
$$

with $\rho_{\sigma}=\rho_{K}$ if $\boldsymbol{u}_{K, \sigma}>0$ and $\rho_{\sigma}=\rho_{L}$ if $\boldsymbol{u}_{K, \sigma}<0$.

The fact that the function $s \in \mathbb{R}_{+}^{*} \rightarrow \ln (s)$ is increasing yields:

$$
\sum_{K \in \mathcal{T}} \ln \left(\rho_{K}\right) \sum_{\sigma=K \mid L}|\sigma| \boldsymbol{u}_{K, \sigma} \rho_{\sigma}+\sum_{K \in \mathcal{T}} \ln \left(\rho_{K}\right) T_{K} \leq 0
$$

Reordering the summations in the second term yields:

$$
\sum_{K \in \mathcal{T}} \ln \left(\rho_{K}\right) T_{K}=\sum_{\sigma=K \mid L} h^{\xi} \frac{|\sigma|}{h_{\sigma}}\left(\rho_{K}+\rho_{L}\right)\left(\ln \left(\rho_{K}\right)-\ln \left(\rho_{L}\right)\right)\left(\rho_{K}-\rho_{L}\right) .
$$


Then, using the mean value theorem, for all $\sigma=K \mid L$ there exists $\tilde{\rho}_{\sigma}$ between $\rho_{K}$ and $\rho_{L}$ such that

$$
\sum_{K \in \mathcal{T}} \ln \left(\rho_{K}\right) T_{K}=\sum_{\sigma=K \mid L} h^{\xi} \frac{|\sigma|}{h_{\sigma}}\left(\rho_{K}-\rho_{L}\right)^{2} \frac{\left(\rho_{K}+\rho_{L}\right)}{\tilde{\rho_{\sigma}}},\left(\tilde{\rho_{\sigma}} \in\left(\rho_{K}, \rho_{L}\right)\right)
$$

and this gives $\sum_{K \in \mathcal{T}} \ln \left(\rho_{K}\right) T_{K} \geq \sum_{\sigma=K \mid L} h^{\xi} \frac{|\sigma|}{h_{\sigma}}\left(\rho_{K}-\rho_{L}\right)^{2}$.

Using this inequality in (3.8) we get

$$
\sum_{K \in \mathcal{T}} \ln \left(\rho_{K}\right) \sum_{\sigma=K \mid L}|\sigma| \boldsymbol{u}_{K, \sigma} \rho_{\sigma}+\sum_{\sigma=K \mid L} h^{\xi} \frac{|\sigma|}{h_{\sigma}}\left(\rho_{K}-\rho_{L}\right)^{2} \leq 0
$$

which be rewritten as

$$
\sum_{\sigma=K \mid L}|\sigma| \boldsymbol{u}_{K, \sigma} \rho_{\sigma}\left(\ln \left(\rho_{K}\right)-\ln \left(\rho_{L}\right)\right)+\sum_{\sigma=K \mid L} h^{\xi} \frac{|\sigma|}{h_{\sigma}}\left(\rho_{K}-\rho_{L}\right)^{2} \leq 0 .
$$

If $\sigma=K \mid L$, we now choose for $K$ the cell satisfying $\boldsymbol{u}_{K, \sigma} \geq 0$. We thus obtain

$$
\sum_{\sigma=K \mid L}|\sigma| \boldsymbol{u}_{K, \sigma} \rho_{K}\left(\ln \left(\rho_{K}\right)-\ln \left(\rho_{L}\right)\right)+\sum_{\sigma=K \mid L} h^{\xi} \frac{|\sigma|}{h_{\sigma}}\left(\rho_{K}-\rho_{L}\right)^{2} \leq 0 .
$$

Adding and substracting the quantity $\sum_{\sigma=K \mid L}|\sigma| \boldsymbol{u}_{K, \sigma}\left(\rho_{K}-\rho_{L}\right)$, we then get

$$
\begin{array}{r}
\sum_{\sigma=K \mid L}|\sigma| \boldsymbol{u}_{K, \sigma}\left[\rho_{K}\left(\ln \left(\rho_{K}\right)-\ln \left(\rho_{L}\right)\right)-\left(\rho_{K}-\rho_{L}\right)\right]+\sum_{\sigma=K \mid L} h^{\xi} \frac{|\sigma|}{h_{\sigma}}\left(\rho_{K}-\rho_{L}\right)^{2} \\
\leq-\sum_{\sigma=K \mid L}|\sigma| \boldsymbol{u}_{K, \sigma}\left(\rho_{K}-\rho_{L}\right)=-\int_{\Omega} \rho \operatorname{div}_{h} \boldsymbol{u} \leq\|\rho\|_{L^{2}(\Omega)}\|\boldsymbol{u}\|_{1, b} .
\end{array}
$$

Since we have $\|\rho\|_{\mathrm{L}^{2}(\Omega)} \leq C$ and $\|\boldsymbol{u}\|_{1, b} \leq C$ where $C$ is given by Proposition 3.2, we obtain

$$
\begin{aligned}
\sum_{\sigma=K \mid L}|\sigma| \boldsymbol{u}_{K, \sigma}[ & \left.\rho_{K}\left(\ln \left(\rho_{K}\right)-\ln \left(\rho_{L}\right)\right)-\left(\rho_{K}-\rho_{L}\right)\right] \\
& +\sum_{\sigma=K \mid L} h^{\xi} \frac{|\sigma|}{h_{\sigma}}\left(\rho_{K}-\rho_{L}\right)^{2} \leq C^{2} .
\end{aligned}
$$

We now use Lemma C.5 with $\psi(s)=\ln (s)$. We obtain the existence for $\sigma=K l L$ of $\tilde{\rho_{\sigma}}$ between $\rho_{K}$ and $\rho_{L}$ such that

$$
\sum_{\sigma=K \mid L}|\sigma| \boldsymbol{u}_{K, \sigma}\left[\rho_{K}\left(\ln \left(\rho_{K}\right)-\ln \left(\rho_{L}\right)\right)-\left(\rho_{K}-\rho_{L}\right)\right]=\sum_{\sigma=K \mid L} \frac{1}{2}|\sigma| u_{K, \sigma}\left(\rho_{K}-\rho_{L}\right)^{2} \tilde{\rho}_{\sigma}^{-1} .
$$

Using this equality in (3.9), we get:

$$
\underbrace{\sum_{\sigma \in \mathcal{E}_{\text {int }}} \frac{1}{2}|\sigma| u_{K, \sigma}\left(\rho_{K}-\rho_{L}\right)^{2} \tilde{\rho}_{\sigma}^{-1}}_{S_{1}}+\underbrace{\sum_{\sigma=K \mid L} h^{\xi} \frac{|\sigma|}{h_{\sigma}}\left(\rho_{K}-\rho_{L}\right)^{2}}_{S_{2}} \leq C^{2} .
$$

This gives $S_{1} \leq C^{2}$ and $S_{2} \leq C^{2}$ and concludes the proof since $S_{2}=h^{\xi}|\rho|_{\mathcal{T}}^{2}$ and $E(\rho) \leq S_{1}$. 


\subsection{Passing to the limit in the discrete problem.}

Theorem 3.4. Let $\alpha>0$ and $0<\xi<2$. Let a sequence of triangulations $\left(\mathcal{T}_{n}\right)_{n \in \mathbb{N}}$ of $\Omega$ be given. We assume that $h_{n}$ (given by $\mathcal{T}_{n}$ ) tends to zero when $n \rightarrow \infty$. In addition, we assume that the sequence of discretizations is regular, in the sense that $\theta_{n} \geq \theta_{0}>0$ for all $n \in \mathbb{N}$. For $n \in \mathbb{N}$, we denote by $\boldsymbol{W}_{h_{n}}$ and $L_{h_{n}}$ the discrete spaces (for velocity, pressure and density) associated to $\mathcal{T}_{n}$ and by $\left(\boldsymbol{u}_{n}, p_{n}, \rho_{n}\right) \in$ $\boldsymbol{W}_{h_{n}} \times L_{h_{n}} \times L_{h_{n}}$ a corresponding solution to the discrete problem (2.5). Then, up to the extraction of a subsequence, when $n \rightarrow \infty$ :

(1) The sequence $\left(\boldsymbol{u}_{n}\right)_{n \in \mathbb{N}}$ (strongly) converges in $\mathrm{L}^{2}(\Omega)^{d}$ to a limit $\boldsymbol{u} \in \mathrm{H}_{0}^{1}(\Omega)^{d}$ and $\left(p_{n}\right)_{n \in \mathbb{N}}$ and $\left(\rho_{n}\right)_{n \in \mathbb{N}}$ converge weakly in $\mathrm{L}^{2}(\Omega)$ to $p, \rho$ respectively;

(2) $(\boldsymbol{u}, p, \rho)$ is a solution to Problem (1.4).

Furthermore, if $\varphi$ is increasing, the sequences $\left(p_{n}\right)_{n \in \mathbb{N}}$ and $\left(\rho_{n}\right)_{n \in \mathbb{N}}$ converge in $L^{p}(\Omega)$ for $1 \leq p<2$ (up to a subsequence).

Proof. The proof is devided in four steps:

\section{- Step 1. Existence of a limit}

The convergence (up to the extraction of a subsequence) of the sequence $\left(\boldsymbol{u}_{n}, p_{n}, \rho_{n}\right)$ is a consequence of the uniform (with respect to $n$ ) estimates of Proposition 3.2 (applying Theorem 2.2 to each component of $\boldsymbol{u}_{n}$ ). Then (up to an extraction) the sequence $\left(\boldsymbol{u}_{n}\right)_{n \in \mathbb{N}}$ (strongly) converges in $\mathrm{L}^{2}(\Omega)^{d}$ to a limit $\boldsymbol{u} \in \mathrm{H}_{0}^{1}(\Omega)^{d}$ and $\left(p_{n}\right)_{n \in \mathbb{N}}$ and $\left(\rho_{n}\right)_{n \in \mathbb{N}}$ converge weakly in $\mathrm{L}^{2}(\Omega)$ to $p$ and $\rho$.

Since $\rho_{n}>0$ and $\int_{\Omega} \rho_{n} \mathrm{~d} \boldsymbol{x}=M$, we obtain, passing to the limit as $n \rightarrow \infty, \rho \geq 0$ a.e. and $\int_{\Omega} \rho \mathrm{d} \boldsymbol{x}=M$.

We now have to prove that $(u, p)$ satisfies (1.4a) (this is proven in Step 2), that $(u, \rho)$ statisfies (1.4b) (Step 3) and that $p=\varphi(\rho)$ a.e. (Step 4). Step 4 will also gives the strong convergence of $\rho$ and $p$ if $\varphi$ is increasing.

\section{- Step 2. Passing to the limit in (2.5a)}

Let $\psi$ be a function of $C_{c}^{\infty}(\Omega)^{d}$. We denote by $\psi_{n}$ the interpolant of $\psi$ in $\boldsymbol{W}_{h_{n}}$, i.e. $\psi_{n}=r_{h_{n}}(\psi)$. Taking $v=\psi_{n}$ in (2.5a), we obtain:

$$
\begin{aligned}
& \mu \int_{\Omega} \nabla_{h_{n}} \boldsymbol{u}_{n}: \nabla_{h_{n}} \psi_{n} \mathrm{~d} \boldsymbol{x}+\frac{\mu}{3} \int_{\Omega} \operatorname{div}_{h_{n}}\left(\boldsymbol{u}_{n}\right) \operatorname{div}_{h_{n}}\left(\psi_{n}\right) \mathrm{d} \boldsymbol{x} \\
& -\int_{\Omega} p_{n} \operatorname{div}_{h_{n}}\left(\psi_{n}\right) \mathrm{d} \boldsymbol{x}=\int_{\Omega} \boldsymbol{f} \cdot \psi_{n} \mathrm{~d} \boldsymbol{x}+\int_{\Omega} \rho_{n} \boldsymbol{g} \cdot \psi_{n} \mathrm{~d} \boldsymbol{x} .
\end{aligned}
$$

We now write $\int_{\Omega} \boldsymbol{\nabla}_{h_{n}} \boldsymbol{u}_{n}: \boldsymbol{\nabla}_{h_{n}} \psi_{n} \mathrm{~d} \boldsymbol{x}=T_{1}+T_{2}$ with

$$
T_{1}=\int_{\Omega} \boldsymbol{\nabla}_{h_{n}} \boldsymbol{u}_{n}: \nabla_{h_{n}}\left(\psi_{n}-\psi\right) \mathrm{d} \boldsymbol{x} \text { and } T_{2}=\int_{\Omega} \boldsymbol{\nabla}_{h_{n}} \boldsymbol{u}_{n}: \nabla_{h_{n}} \psi \mathrm{d} \boldsymbol{x} .
$$

Using the third property of the interpolation operator given in theorem 2.1, we get, with $c\left(\theta_{0}\right)$ only depending on $\Omega$ and $\theta_{0}$,

$$
\left|T_{1}\right| \leq\left\|\boldsymbol{u}_{n}\right\|_{1, b}\left\|\left(\psi_{n}-\psi\right)\right\|_{1, b} \leq c\left(\theta_{0}\right) h_{n}\left\|\boldsymbol{u}_{n}\right\|_{1, b}|\psi|_{\mathrm{H}^{2}(\Omega)}
$$

and thus $T_{1}$ tends to zero as $\mathrm{n}$ tends to $+\infty$. Integrating by parts over each control volume, the term $T_{2}$ reads:

$$
T_{2}=-\int_{\Omega} \boldsymbol{u}_{n} \cdot \Delta \psi \mathrm{d} \boldsymbol{x}+\sum_{\sigma \in \mathcal{E}_{\text {int }}} \int_{\sigma}\left[\boldsymbol{u}_{n}\right]_{\sigma}: \nabla \psi \mathrm{d} \gamma
$$


where $\left[\boldsymbol{u}_{n}\right]_{\sigma}=\left(\boldsymbol{u}_{K} \otimes \boldsymbol{n}_{K}+\boldsymbol{u}_{L} \otimes \boldsymbol{n}_{L}\right.$ ) if $\sigma=K \mid L$ (for all $K \in \mathcal{T}_{n}, \boldsymbol{u}_{K}$ is the value of $\boldsymbol{u}_{n}$ in $K$, and $\boldsymbol{n}_{K}$ is the normal vector to $\partial K$ exterior to $K$ ). We omit the dependance of $\mathcal{E}_{\text {int }}$ with respect to $n$. Noticing that $\boldsymbol{n}_{L}=-\boldsymbol{n}_{K}$ and applying Lemma 2.4 in [10], we get, again with $c\left(\theta_{0}\right)$ only depending on $\Omega$ and $\theta_{0}$,

$$
\left|\sum_{\sigma \in \mathcal{E}_{\text {int }}} \int_{\sigma}\left[\boldsymbol{u}_{n}\right]: \nabla \psi n_{\sigma} \mathrm{d} \gamma\right| \leq c\left(\theta_{0}\right) h_{n}\left\|\boldsymbol{u}_{n}\right\|_{1, b}|\psi|_{\mathrm{H}^{2}(\Omega)}
$$

and thus tends to zero as $\mathrm{n}$ tends to $+\infty$. On the other hand we have:

$$
\begin{gathered}
-\int_{\Omega} \boldsymbol{u}_{n} \cdot \Delta \psi \mathrm{d} \boldsymbol{x} \rightarrow-\int_{\Omega} \boldsymbol{u} \cdot \Delta \psi \mathrm{d} \boldsymbol{x} \text { as } n \rightarrow+\infty \\
=\int_{\Omega} \boldsymbol{\nabla} \boldsymbol{u}: \boldsymbol{\nabla} \psi \mathrm{d} \boldsymbol{x} \text { since } \boldsymbol{u} \in \mathrm{H}_{0}^{1}(\Omega) .
\end{gathered}
$$

Then, the first term of the Left Hand Side of (3.10) converges to $\int_{\Omega} \boldsymbol{\nabla} \boldsymbol{u}: \boldsymbol{\nabla} \psi \mathrm{d} \boldsymbol{x}$ as $n \rightarrow \infty$. For the second term of (3.10), using the first property of the interpolation operator in theorem 2.1, we get, with $\left[\boldsymbol{u}_{n} \cdot \boldsymbol{n}\right]_{\sigma}=\boldsymbol{u}_{K} \cdot \boldsymbol{n}_{K}+\boldsymbol{u}_{L} \cdot \boldsymbol{n}_{L}$,

$$
\begin{aligned}
& \int_{\Omega} \operatorname{div}_{h_{n}}\left(\boldsymbol{u}_{n}\right) \operatorname{div}_{h_{n}}\left(\psi_{n}\right) \mathrm{d} \boldsymbol{x}=\int_{\Omega} \operatorname{div}_{h_{n}}\left(\boldsymbol{u}_{n}\right) \operatorname{div}(\psi) \mathrm{d} \boldsymbol{x} \\
& =\sum_{K \in \mathcal{T}_{n}} \sum_{i \leq d} \sum_{j \leq d} \int_{K}\left(\boldsymbol{u}_{n}\right)_{i} \frac{\partial^{2} \psi_{j}}{\partial x_{i} \partial x_{j}} \mathrm{~d} \boldsymbol{x}+\sum_{K \in \mathcal{T}_{n}} \int_{\partial K} \boldsymbol{u}_{n} \operatorname{div} \psi \cdot n_{K} \mathrm{~d} \gamma \\
& =\sum_{K \in \mathcal{T}_{n}} \sum_{i \leq d} \sum_{j \leq d} \int_{K}\left(\boldsymbol{u}_{n}\right)_{i} \frac{\partial^{2} \psi_{j}}{\partial x_{i} \partial x_{j}} \mathrm{~d} \boldsymbol{x}+\sum_{\sigma \in \mathcal{E}_{\text {int }}} \int_{\sigma}\left[\boldsymbol{u}_{n} \cdot \boldsymbol{n}\right]_{\sigma} \operatorname{div} \psi \mathrm{d} \gamma \\
& =T_{2,1}+T_{2,2} .
\end{aligned}
$$

Applying Lemma 2.4 in [10], we get, with $c\left(\theta_{0}\right)$ only depending on $\Omega$ and $\theta_{0}$,

$$
\left|T_{2,2}\right|=\left|\sum_{\sigma \in \mathcal{E}_{\text {int }}} \int_{\sigma}\left[\boldsymbol{u}_{n} \cdot \boldsymbol{n}\right]_{\sigma} \operatorname{div} \psi \mathrm{d} \gamma\right| \leq c\left(\theta_{0}\right) h_{n}\left\|\boldsymbol{u}_{n}\right\|_{1, b}|\operatorname{div} \psi|_{\mathrm{H}^{1}(\Omega)}
$$

and thus $T_{2,2}$ tends to zero as $n$ tends to $+\infty$. Then, the second term of (3.10) has the same limit as $T_{2,1}$ and this limit is $\int_{\Omega} \operatorname{div} \boldsymbol{u} \operatorname{div} \psi \mathrm{d} \boldsymbol{x}$.

For the third term of (3.10), we use, once again, Theorem 2.1 which yields:

$$
\int_{\Omega} p_{n} \operatorname{div}_{h_{n}}\left(\psi_{n}\right) \mathrm{d} \boldsymbol{x}=\int_{\Omega} p_{n} \operatorname{div}(\psi) \mathrm{d} \boldsymbol{x} \rightarrow \int_{\Omega} p \operatorname{div}(\psi) \mathrm{d} \boldsymbol{x} \text { as } n \rightarrow+\infty .
$$

We now consider the Right Hand Side of (3.10). Since $\psi_{n} \rightarrow \psi$ in $L^{2}(\Omega)^{d}$ we obtain

$$
\int_{\Omega} \boldsymbol{f} \cdot \psi_{n} \mathrm{~d} \boldsymbol{x} \rightarrow \int_{\Omega} \boldsymbol{f} \cdot \psi \mathrm{d} \boldsymbol{x} \text { as } n \rightarrow+\infty .
$$

For the last term of (3.10), we use, once again, the $\left(L^{2}\right)^{d}$ convergence of $\psi_{n}$ to $\psi$ and we use the weak- $L^{2}$ convergence of $\rho_{n}$ to $\rho$. We obtain

$$
\int_{\Omega} \rho_{n} \boldsymbol{g} \cdot \psi_{n} \mathrm{~d} \boldsymbol{x} \rightarrow \int_{\Omega} \rho \boldsymbol{g} \cdot \psi \mathrm{d} \boldsymbol{x} \text { as } n \rightarrow+\infty .
$$

Finally, we can pass to limit in (3.10) as $n \rightarrow \infty$ and we get (1.4a) for all $\psi \in$ $C_{c}^{\infty}(\Omega)^{d}$ (and then, by density, for all $\left.\psi \in H_{0}^{1}(\Omega)^{d}\right)$, namely:

$$
\begin{array}{r}
\mu \int_{\Omega} \boldsymbol{\nabla} \boldsymbol{u}: \nabla \psi \mathrm{d} \boldsymbol{x}+\frac{\mu}{3} \int_{\Omega} \operatorname{div}(\boldsymbol{u}) \operatorname{div}(\psi) \mathrm{d} \boldsymbol{x}-\int_{\Omega} p \operatorname{div}(\psi) \mathrm{d} \boldsymbol{x} \\
=\int_{\Omega} \boldsymbol{f} \cdot \psi \mathrm{d} \boldsymbol{x}+\int_{\Omega} \rho \boldsymbol{g} \cdot \psi \mathrm{d} \boldsymbol{x} .
\end{array}
$$




\section{- Step 3. Passing to the limit in (2.5b)}

Let $\psi$ be a function of $C_{c}^{\infty}(\Omega)^{d}$.Multiplying $(2.5 \mathrm{~b})$ by $\psi_{K}=\psi\left(x_{K}\right)$ and summing over $K \in \mathcal{T}_{n}$ we obtain:

$$
\begin{aligned}
T_{1}+T_{2}+T_{3}= & \sum_{K \in \mathcal{T}_{n}} \psi_{K} \sum_{\sigma \in \mathcal{E}(K)}|\sigma| \boldsymbol{u}_{K, \sigma} \rho_{\sigma}+\sum_{K \in \mathcal{T}_{n}} h_{n}^{\alpha}|K| \psi_{K}\left(\rho_{K}-\rho^{*}\right) \\
& +\sum_{K \in \mathcal{T}_{n}} \psi_{K} \sum_{\sigma \in \mathcal{E}(K)} h_{n}^{\xi} \frac{|\sigma|}{h_{\sigma}}\left(\rho_{K}+\rho_{L}\right)\left(\rho_{K}-\rho_{L}\right)=0 .
\end{aligned}
$$

The first term $T_{1}$ reads, with $\psi_{\sigma}=\psi\left(x_{\sigma}\right)$,

$$
\begin{gathered}
T_{1}=\sum_{K \in \mathcal{T}_{n}} \sum_{\sigma \in \mathcal{E}(K)}|\sigma| \boldsymbol{u}_{K, \sigma} \rho_{\sigma} \psi_{K}=\sum_{K \in \mathcal{T}_{n}} \sum_{\sigma \in \mathcal{E}(K)}|\sigma| \boldsymbol{u}_{K, \sigma} \rho_{\sigma}\left(\psi_{K}-\psi_{\sigma}\right) \\
=\sum_{K \in \mathcal{T}_{n}} \sum_{\sigma \in \mathcal{E}(K)}|\sigma| \boldsymbol{u}_{K, \sigma} \rho_{K}\left(\psi_{K}-\psi_{\sigma}\right)+R_{1} \\
\text { with } R_{1}=-\sum_{K \in \mathcal{T}_{n}} \sum_{\sigma \in \mathcal{E}(K)}|\sigma| \boldsymbol{u}_{K, \sigma}\left(\rho_{K}-\rho_{\sigma}\right)\left(\psi_{K}-\psi_{\sigma}\right) .
\end{gathered}
$$

Then,

$$
\begin{gathered}
T_{1}=\sum_{K \in \mathcal{T}_{n}} \rho_{K} \psi_{K} \sum_{\sigma \in \mathcal{E}(K)}|\sigma| \boldsymbol{u}_{K, \sigma}-\sum_{K \in \mathcal{T}_{n}} \rho_{K} \sum_{\sigma \in \mathcal{E}(K)}|\sigma| \boldsymbol{u}_{K, \sigma} \psi_{\sigma}+R_{1} \\
=\sum_{K \in \mathcal{T}_{n}} \rho_{K} \int_{K} \psi \operatorname{div} u_{n} \mathrm{~d} \boldsymbol{x}-\sum_{K \in \mathcal{T}_{n}} \rho_{K} \int_{K} \operatorname{div}\left(\psi u_{n}\right) \mathrm{d} \boldsymbol{x}+R_{1}+R_{2}+R_{3} . \\
\text { with } R_{2}=-\sum_{K \in \mathcal{T}_{n}} \rho_{K} \int_{K}\left(\psi-\psi_{K}\right) \operatorname{div} \boldsymbol{u}_{n} \mathrm{~d} \boldsymbol{x} \\
\text { and } R_{3}=\sum_{K \in \mathcal{T}_{n}} \rho_{K} \sum_{\sigma \in \mathcal{E}(K)} \int_{\sigma}\left(\psi-\psi_{\sigma}\right) \boldsymbol{u}_{n} \cdot \boldsymbol{n}_{K, \sigma} \mathrm{d} \gamma \\
T_{1}=-\int_{\Omega} \rho_{n} \boldsymbol{u}_{n} \cdot \boldsymbol{\nabla} \psi+R_{1}+R_{2}+R_{3} .
\end{gathered}
$$

Let us now prove that the terms $R_{1}, R_{2}, R_{3} \rightarrow 0$ as $\mathrm{n} \rightarrow+\infty$. We begin with $R_{1}$.

One has, with $C_{\psi}=\||\nabla \psi|\|_{L^{\infty}(\Omega)}$,

$$
\begin{array}{r}
\left|R_{1}\right|=\left|\sum_{K \in \mathcal{T}_{n}} \sum_{\sigma \in \mathcal{E}(K)}\right| \sigma\left|\boldsymbol{u}_{K, \sigma}\left(\rho_{K}-\rho_{\sigma}\right)\left(\psi_{K}-\psi_{\sigma}\right)\right| \\
\leq C_{\psi} \sum_{\sigma=K \mid L}\left(h_{K}+h_{L}\right)\left|\rho_{K}-\rho_{L}\right||\sigma|\left|\boldsymbol{u}_{K, \sigma}\right| .
\end{array}
$$

This gives, with the Cauchy-Schwarz inequality,

$$
\left|R_{1}\right| \leq C_{\psi} E\left(\rho_{n}\right)\left(\sum_{\sigma=K \mid L} \frac{\left(h_{K}+h_{L}\right)^{2}}{\min \left(\frac{1}{\rho_{K}}, \frac{1}{\rho_{L}}\right)}|\sigma|\left|\boldsymbol{u}_{K, \sigma}\right|\right)^{\frac{1}{2}} .
$$

Then,

$$
\left|R_{1}\right| \leq C_{\psi} E\left(\rho_{n}\right)(\underbrace{\left.\sum_{\sigma=K \mid L}\left(h_{K}+h_{L}\right)^{2}\left(\rho_{K}+\rho_{L}\right)|\sigma|\left|\boldsymbol{u}_{K, \sigma}\right|\right)^{\frac{1}{2}}}_{S 2} .
$$


Using again the Cauchy Schwarz inequality we thus obtain:

$$
S_{2} \leq\left(\sum_{\sigma=K \mid L}\left(h_{K}+h_{L}\right)|\sigma|\left(\rho_{K}+\rho_{L}\right)^{2}\right)^{1 / 2}\left(\sum_{\sigma=K \mid L}\left(h_{K}+h_{L}\right)^{3}\left|\sigma \| \boldsymbol{u}_{K, \sigma}\right|^{2}\right)^{1 / 2}
$$

The properties of the scheme given in section 2 and Hölder's Inequality yields, with $C_{1}\left(\theta_{0}\right)$ and $C_{2}\left(\theta_{0}\right)$ only depending on $\Omega$ and $\theta_{0}$,

$$
\begin{aligned}
& S_{2} \leq C_{1}\left(\theta_{0}\right)(\left.\sum_{\sigma=K \mid L}(|K|+|L|)\left(\rho_{K}+\rho_{L}\right)^{2}\right)^{1 / 2}\left(\sum_{\sigma=K \mid L}\left(h_{K}+h_{L}\right)^{3}\left\|\boldsymbol{u}_{n}\right\|_{L^{2}(\sigma)}^{2}\right)^{1 / 2} \\
& \leq C_{2}\left(\theta_{0}\right)\left(\sum_{K \in \mathcal{T}_{n}}|K| \rho_{K}^{2}\right)^{1 / 2}\left(\sum_{\sigma=K \mid L} h_{\sigma}^{3}\left\|\boldsymbol{u}_{n}\right\|_{L^{2}(\sigma)}^{2}\right)^{1 / 2} .
\end{aligned}
$$

The estimate on $\rho_{n}$ in $L^{2}(\Omega)$ gives the existence of $C_{3}$, only depending on the $L^{2}$-bound on $\rho_{n}$ and on $C_{2}\left(\theta_{0}\right)$ such that:

$$
S_{2} \leq C_{3}\left(\sum_{\sigma=K \mid L} h_{\sigma}^{3}\left\|\boldsymbol{u}_{n}\right\|_{L^{2}(\sigma)}^{2}\right)^{1 / 2}
$$

By Lemma 2.3 in [10], we have:

$$
\left\|\boldsymbol{u}_{n}\right\|_{L^{2}(\sigma)} \leq\left(d \frac{|\sigma|}{|K|}\right)^{1 / 2}\left(\left\|\boldsymbol{u}_{n}\right\|_{L^{2}(K)}+h_{K}\left\|\nabla \boldsymbol{u}_{n}\right\|_{L^{2}(K)}\right) .
$$

We thus obtain, with some $C_{4}$ and $C_{5}$ only depending on the $L^{2}$-bound on $\rho_{n}, \Omega$ and $\theta_{0}$,

$$
\begin{gathered}
S_{2} \leq C_{4}\left(\sum_{K \in \mathcal{T}_{n}} h_{K}^{2}\left(\left\|\boldsymbol{u}_{n}\right\|_{L^{2}(K)}^{2}+h_{K}^{2}\left\|\nabla \boldsymbol{u}_{n}\right\|_{L^{2}(K)}^{2}\right)\right)^{1 / 2} \\
\leq C_{5} h_{n}\left(\left\|\boldsymbol{u}_{n}\right\|_{L^{2}(\Omega)}^{2}+\left\|\boldsymbol{u}_{n}\right\|_{1, b}^{2}\right)^{1 / 2}
\end{gathered}
$$

Finally, thanks to the bound on $u_{n}$ (Proposition 3.2) we get $\lim _{n \rightarrow \infty} S_{2}=0$ and thanks to the bound on $E\left(\rho_{n}\right)$ (Lemma 3.3) we conclude that $\lim _{n \rightarrow \infty} R_{1}=0$.

We now come to $R_{2}$. One has

$$
\left|R_{2}\right| \leq C_{\psi} h_{n}\left\|\rho_{n}\right\|_{\mathrm{L}^{2}(\Omega)}\left\|\operatorname{div}_{h_{n}}\left(\boldsymbol{u}_{n}\right)\right\|_{\mathrm{L}^{2}(\Omega)} \leq C_{\psi} h_{n}\left\|\rho_{n}\right\|_{\mathrm{L}^{2}(\Omega)}\left\|\boldsymbol{u}_{n}\right\|_{1, b},
$$

which tends to 0 as $n \rightarrow+\infty$.

It remains to treat $R_{3}$. One has

$$
\begin{aligned}
\left|R_{3}\right| & =\left|\sum_{K \in \mathcal{T}_{n}} \rho_{K} \sum_{\sigma \in \mathcal{E}(K)} \int_{\sigma}\left(\psi-\psi_{\sigma}\right) \boldsymbol{u}_{n} \cdot \boldsymbol{n}_{K, \sigma} \mathrm{d} \gamma\right| \\
& =\left|\sum_{\sigma=K \mid L}\left(\rho_{K}-\rho_{L}\right) \int_{\sigma}\left(\psi-\psi_{\sigma}\right) \boldsymbol{u}_{n} \cdot \boldsymbol{n}_{K, \sigma} \mathrm{d} \gamma\right|
\end{aligned}
$$

In order to prove that $\lim _{n \rightarrow \infty} R_{3}=0$, we treat separetely, the interfaces $\sigma$ where the sign of $\boldsymbol{u}_{n} \cdot \boldsymbol{n}_{K, \sigma}$ is constant or not (for $\sigma$ between $K$ and $L$, it can be different for $K$ and $L$ ). For the interfaces where the sign of $\boldsymbol{u}_{n} \cdot \boldsymbol{n}_{K, \sigma}$ is constant, we use the same arguments as for the first term $R_{1}$ (bound on $\boldsymbol{u}_{n}$ and bound on $E\left(\rho_{n}\right)$ ) and we get a bound in $\sqrt{h_{n}}$ for the sum of these terms. For the interfaces where the sign of $\boldsymbol{u}_{n} \cdot \boldsymbol{n}_{K, \sigma}$ is not constant, we use a bound (only depending of the regularity of the mesh, that is $\theta$ ) of $\left\|\boldsymbol{u}_{n} \cdot \boldsymbol{n}_{K, \sigma}\right\|_{L^{1}(\sigma)}$ by $\left\|\boldsymbol{\nabla} \boldsymbol{u}_{n}\right\|_{L^{1}(K)}$ (this bound uses the fact 
that $\boldsymbol{u}_{n} \cdot \boldsymbol{n}_{K, \sigma}$ vanishes at a point of $\left.\sigma\right)$. Then, thanks to the bound on $\left\|\boldsymbol{u}_{n}\right\|_{1, b}$ and $\left\|\rho_{n}\right\|_{L^{2}(\Omega)}$, we get a bound in $h_{n}$ for the sum of these terms.

Finally, since $\lim _{n \rightarrow \infty} R_{i}=0$ for $i=1,2,3$, one has

$$
\lim _{n \rightarrow \infty} T_{1}=-\lim _{n \rightarrow \infty} \int_{\Omega} \rho_{n} u_{n} \cdot \nabla \psi \mathrm{d} \boldsymbol{x}
$$

Using the $L^{2}(\Omega)$ convergence of $\boldsymbol{u}_{n}$ and the $L^{2}(\Omega)$-weak convergence of $\rho_{n}$, we conclude that

$$
\lim _{n \rightarrow \infty} T_{1}=-\int_{\Omega} \rho \boldsymbol{u} \cdot \nabla \psi \mathrm{d} \boldsymbol{x} .
$$

We now prove that $T_{2}$ and $T_{3}$ tend to 0 as $n \rightarrow \infty$. We remark that

$$
\left|T_{2}\right|=\left|\sum_{K \in \mathcal{T}_{n}} h_{K}^{\alpha}\right| K\left|\left(\rho_{K}-\rho^{*}\right) \psi_{K}\right| \leq h_{n}^{\alpha} 2 M\|\psi\|_{L^{\infty}(\Omega)} \rightarrow 0 \text { as } n \rightarrow+\infty
$$

and

$$
\begin{array}{r}
\left|T_{3}\right|=\left|\sum_{K \in \mathcal{T}_{n}} \sum_{\sigma \in \mathcal{E}(K)} h_{n}^{\xi} \frac{|\sigma|}{h_{\sigma}}\left(\rho_{K}+\rho_{L}\right)\left(\rho_{K}-\rho_{L}\right) \psi_{K}\right| \\
=\left|h_{n}^{\xi} \sum_{\sigma=K \mid L} \frac{|\sigma|}{h_{\sigma}}\left(\rho_{K}+\rho_{L}\right)\left(\rho_{K}-\rho_{L}\right)\left(\psi_{K}-\psi_{L}\right)\right| \\
\leq C_{\psi} h_{n}^{\xi} \sum_{\sigma=K \mid L}\left(h_{K}+h_{L}\right) \frac{|\sigma|}{h_{\sigma}}\left(\rho_{K}+\rho_{L}\right)\left|\rho_{K}-\rho_{L}\right| .
\end{array}
$$

We now use the Cauchy-Schwarz Inequality to obtain, with $C_{1}$ only depending on $\psi$ and the bound on $h_{n}^{\xi}\left|\rho_{n}\right|^{2}$ given by Lemma 3.3,

$$
\begin{array}{r}
\left|T_{3}\right| \leq C_{\psi} h_{n}^{\xi}\left|\rho_{n}\right| \mathcal{T}\left(\sum_{\sigma=K \mid L}\left(h_{K}+h_{L}\right)^{2} \frac{|\sigma|}{h_{\sigma}}\left(\rho_{K}+\rho_{L}\right)^{2}\right)^{\frac{1}{2}} \\
\leq C_{1} h_{n}^{\xi / 2}\left(\sum_{\sigma=K \mid L}\left(h_{K}+h_{L}\right)^{2} \frac{|\sigma|}{h_{\sigma}}\left(\rho_{K}+\rho_{L}\right)^{2}\right)^{\frac{1}{2}} .
\end{array}
$$

The properties of the mesh given in section 2 yield the existence of $c\left(\theta_{0}\right)$ only depending on $\Omega$ and $\theta_{0}$ such that

$$
\frac{|\sigma|}{h_{\sigma}} \leq c\left(\theta_{0}\right) \frac{|K|+|L|}{\left(h_{K}+h_{L}\right)^{2}} .
$$

We thus obtain $\left|T_{3}\right| \leq C_{1} \sqrt{c\left(\theta_{0}\right)} h_{n}^{\xi / 2}\left(\sum_{\sigma=K \mid L}(|K|+|L|)\left(\rho_{K}+\rho_{L}\right)^{2}\right)^{\frac{1}{2}}$. Thanks to the $L^{2}$-estimate on $\rho_{n}$, we then conclude that $\lim _{n \rightarrow \infty} T_{3}=0$.

Finally, we can pass to the limit in (3.11) as $n \rightarrow \infty$ and we obtain (1.4b) for all $\psi \in C_{c}^{\infty}(\Omega)$. This gives also (1.4b) for all $\psi \in W^{1, \infty}(\Omega)$ thanks to Lemma B.6 (since $u \in H_{0}^{1}(\Omega)$ and $\rho \in L^{2}(\Omega)$ ).

\section{- Step 4. Passing to the limit in the Equation Of State}

In order to conclude the proof of Theorem 3.4, it remains to prove that the equation of state is satisfied, that is $p=\varphi(\rho)$ a.e. in $\Omega$. This is a tricky part of the proof.

Let $\left(q_{n}\right)_{n \in \mathbb{N}}$ be a sequence such that $q_{n} \in L_{h_{n}}$ for all $n \in \mathbb{N}$. We assume that the sequence $\left(q_{n}\right)_{n \in \mathbb{N}}$ weakly converges in $\mathrm{L}^{2}(\Omega)$ to $q \in \mathrm{L}^{2}(\Omega)$ and satisfies

$$
\left|q_{n}\right|_{\mathcal{T}} \leq c h_{n}^{-\eta}
$$


where $c$ is a positive real number and $\eta$ is such that $\eta<1$. Then one has:

$$
\forall \psi \in \mathrm{C}_{c}^{\infty}(\Omega), \quad \lim _{n \rightarrow \infty} \int_{\Omega}\left(\operatorname{div}_{h_{n}}\left(\boldsymbol{u}_{n}\right)-p_{n}\right) q_{n} \psi \mathrm{d} \boldsymbol{x}=\int_{\Omega}(\operatorname{div}(\boldsymbol{u})-p) q \psi \mathrm{d} \boldsymbol{x} .
$$

This result is proven in [6], Proposition 5.9. Indeed, in Proposition 5.9 of [6] the hypothesis on $\rho$ is $\rho \in L^{2 \gamma}(\Omega), \gamma>1$, and the sequence $\left(\rho_{n}\right)_{n \in \mathbb{N}}$ converges to $\rho$ weakly in $L^{2 \gamma}(\Omega)$, but the proof given in [6] is also true for $\gamma=1$.

Taking $q_{n}=\rho_{n}$ in (3.12) (which is possible with $\eta=\xi / 2$, thanks to Lemma 3.3), one obtains

$$
\forall \psi \in \mathrm{C}_{c}^{\infty}(\Omega), \quad \lim _{n \rightarrow \infty} \int_{\Omega}\left(\operatorname{div}_{h_{n}}\left(\boldsymbol{u}_{n}\right)-p_{n}\right) \rho_{n} \psi \mathrm{d} \boldsymbol{x}=\int_{\Omega}(\operatorname{div}(\boldsymbol{u})-p) \rho \psi \mathrm{d} \boldsymbol{x}
$$

We now want to prove $(3.13)$ with $\psi=1$ a.e. on $\Omega$. This is possible, thanks to Lemma C.1, if the sequence $\left(\left(\operatorname{div}_{h_{n}} \boldsymbol{u}_{n}-p_{n}\right) \rho_{n}\right)_{n \in \mathbb{N}}$ is equi-integrable. The condition (1.1) on $\varphi$, and the $L^{2}$-bound on $\operatorname{div}_{h_{n}} \boldsymbol{u}_{n}$ and on $p_{n}$ will give this equi-integrability. Let $a>0$ and $b>0$ given by (1.1). One has a.e. on $\Omega$,

$$
a \rho_{n} \leq \varphi\left(\rho_{n}\right)+b=p_{n}+b,
$$

so that

$$
\rho_{n}^{2} \leq \frac{2 p_{n}^{2}}{a^{2}}+\frac{2 b^{2}}{a^{2}}
$$

If $C$ is a bound for the $L^{2}$-norm of $p_{n}$ (such a bound is given by Proposition 3.2), one obtains for any borelian subset $A$ of $\Omega$,

$$
\int_{A} \rho_{n}^{2} d x \leq \frac{2 C^{2}}{a^{2}}+\frac{2 b^{2}}{a^{2}}|A| .
$$

Let $\varepsilon>0$, we then take $a^{2}=2 C^{2} / \varepsilon$ which yields:

$$
\int_{A} \rho_{n}^{2} d x \leq \varepsilon+\frac{2 b^{2}}{a^{2}}|A| .
$$

and then, with $\delta=\frac{\varepsilon a^{2}}{2 b^{2}}$,

$$
|A| \leq \delta \Rightarrow \int_{A} \rho_{n}^{2} d x \leq 2 \varepsilon
$$

This proves the equi-integrability of the sequence $\left(\rho_{n}^{2}\right)_{n \in \mathbb{N}}$. Since the sequence $\left(\left(\operatorname{div}_{h_{n}} \boldsymbol{u}_{n}-p_{n}\right)\right)_{n \in \mathbb{N}}$ is bounded in $\mathrm{L}^{2}(\Omega)$, we then easily conclude (with the CauchyScwarz inequality) that the sequence $\left(\left(\operatorname{div}_{h_{n}} \boldsymbol{u}_{n}-p_{n}\right) \rho_{n}\right)_{n \in \mathbb{N}}$ is equi-integrable. Thus Lemma C.1 yields the conclusion, namely $(3.13)$ is true for $\psi=1$ a.e. on $\Omega$ :

$$
\lim _{n \rightarrow \infty} \int_{\Omega}\left(\operatorname{div}_{h_{n}}\left(\boldsymbol{u}_{n}\right)-p_{n}\right) \rho_{n} \mathrm{~d} \boldsymbol{x}=\int_{\Omega}(\operatorname{div}(\boldsymbol{u})-p) \rho \mathrm{d} \boldsymbol{x} .
$$

We now want to get rid of $\int_{\Omega} \rho \operatorname{div}(\boldsymbol{u}) \mathrm{d} \boldsymbol{x}$ and $\int_{\Omega} \rho_{n} \operatorname{div}\left(\boldsymbol{u}_{n}\right) \mathrm{d} \boldsymbol{x}$ in (3.14).

Since $\rho \in L^{2}(\Omega), \rho \geq 0$ a.e. in $\Omega, u \in H_{0}^{1}(\Omega)^{d}$ and $(\rho, u)$ satisfies $(1.4 \mathrm{~b})$, we can use Lemma B.1. It gives

$$
\int_{\Omega} \rho \operatorname{div}(\boldsymbol{u}) \mathrm{d} \boldsymbol{x}=0
$$


Then, using (3.15) in (3.14) we get:

$$
\lim _{n \rightarrow \infty} \int_{\Omega}\left(p_{n}-\operatorname{div}_{h_{n}}\left(\boldsymbol{u}_{n}\right)\right) \rho_{n} \mathrm{~d} \boldsymbol{x}-\int_{\Omega} p \rho \mathrm{d} \boldsymbol{x}=0 .
$$

By Lemma 3.1 we also have $\int_{\Omega} \rho_{n} \operatorname{div}_{h_{n}}\left(\boldsymbol{u}_{n}\right) \mathrm{d} \boldsymbol{x} \leq 0$. Hence:

$$
\limsup _{n \rightarrow \infty} \int_{\Omega} p_{n} \rho_{n} \mathrm{~d} \boldsymbol{x} \leq \int_{\Omega} p \rho \mathrm{d} \boldsymbol{x} .
$$

To conclude the proof of $p=\varphi(\rho)$, we will now use the so called Minty trick. Let $\bar{\rho} \in L^{2}(\Omega)$ such that $\varphi(\bar{\rho}) \in L^{2}(\Omega)$. We define for $n \in \mathbb{N}$ the function $G_{n}$ by

$$
G_{n}=\left(\varphi\left(\rho_{n}\right)-\varphi(\bar{\rho})\right)\left(\rho_{n}-\bar{\rho}\right)=\left(p_{n}-\varphi(\bar{\rho})\right)\left(\rho_{n}-\bar{\rho}\right) .
$$

One has $G_{n} \in L^{1}(\Omega), G_{n} \geq 0$ a.e. in $\Omega$ (since $\varphi$ is nondecreasing) and

$$
0 \leq \int_{\Omega} G_{n} d x=\int_{\Omega}\left(p_{n} \rho_{n}-p_{n} \bar{\rho}-\varphi(\bar{\rho}) \rho_{n}+\varphi(\bar{\rho}) \bar{\rho}\right) \mathrm{d} \boldsymbol{x} .
$$

Using (3.16) and the weak convergences of $p_{n}$ to $p$ and $\rho_{n}$ to $\rho$ in $L^{2}(\Omega)$, we obtain:

$$
0 \leq \limsup _{n \rightarrow \infty} \int_{\Omega} G_{n} d x \leq \int_{\Omega}(p-\varphi(\bar{\rho}))(\rho-\bar{\rho}) \mathrm{d} \boldsymbol{x} .
$$

We have thus proven that for all $\bar{\rho} \in L^{2}(\Omega)$ such that $\varphi(\bar{\rho}) \in L^{2}(\Omega)$ one has

$$
\int_{\Omega}(p-\varphi(\bar{\rho}))(\rho-\bar{\rho}) \mathrm{d} \boldsymbol{x} \geq 0 .
$$

We now have to choose $\bar{\rho}$ conveniently to deduce $p=\varphi(\rho)$ a.e. on $\Omega$ from (3.18). The idea of the Minty trick is to take $\bar{\rho}=\rho+(1 / k) \psi$ with $\psi \in C_{c}^{\infty}(\Omega), k \in \mathbb{N}^{\star}$ and to let $k$ goes to $+\infty$. Unfortunately, $\varphi(\rho+(1 / k) \psi)$ is not necessarily in $L^{2}(\Omega)$. then, such a choice for $\bar{\rho}$ is not possible. We will use here (and only here) the convexity of $\varphi$. Since $\left(\rho_{n}\right)_{n}$ weakly converges in $L^{2}(\Omega)$ to $\rho$ and since the sequence $\left(\varphi\left(\rho_{n}\right)\right)_{n \in \mathbb{N}}$ is bounded in $L^{2}(\Omega)$, we deduce, using the convexity of $\varphi$, that $\varphi(\rho) \in L^{2}(\Omega)$. This is proven in Lemma B.8. This allows us a convenient choice for $\bar{\rho}$.

Let $\psi \in C_{c}^{\infty}(\Omega, \mathbb{R})$. For $k, m \in \mathbb{N}^{\star}$, we set

$$
\rho_{k, m}=\rho+\frac{1}{k} \psi 1_{\rho \leq m} .
$$

Since $\rho \in L^{2}(\Omega)$, one has $\rho_{k, m} \in L^{2}(\Omega)$. Using the fact that $\varphi$ is nondecreasing (and nonnegative), we have, with $M=\|\psi\|_{L^{\infty}(\Omega)}$,

$$
\varphi\left(\rho_{k, m}\right) \leq \varphi(\rho)+\varphi(m+M),
$$

so that $\varphi\left(\rho_{k, m}\right) \in L^{2}(\Omega)$ (since $\varphi(\rho) \in L^{2}(\Omega)$ ). Then, since $\rho_{k, m}$ and $\varphi\left(\rho_{k, m}\right)$ belong to $L^{2}(\Omega)$, we can choose $\bar{\rho}=\rho_{k, m}$ in (3.18). We obtain

$$
\int_{\Omega}\left(p-\varphi\left(\rho+\frac{1}{k} \psi 1_{\rho \leq m}\right)\right) \psi 1_{\rho \leq m} \leq 0 .
$$

Fixing $m$ in $\mathbb{N}^{\star}$, we use the Dominated Convergence theorem on the sequence $\left(g_{k}\right)_{k \in \mathbb{N}^{\star}}$ with $g_{k}=\left(p-\varphi\left(\rho+\frac{1}{k} \psi 1_{\rho \leq m}\right)\right) \psi 1_{\rho \leq m}$. Indeed, the continuity of $\varphi$ gives $g_{k} \rightarrow(p-\varphi(\rho)) \psi 1_{\rho \leq m}$ a.e. in $\Omega$. Furthermore, since $\varphi$ is nondecreasing, one has, for all $n \in \mathbb{N}^{\star}$,

$$
\left|g_{k}\right| \leq H=[p+\varphi(\rho)+\varphi(m+M)]|\psi| \text { a.e. in } \Omega,
$$


and $H \in L^{1}(\Omega)$. Then, the Dominated Convergence theorem yields

$$
\int_{\Omega}(p-\varphi(\rho)) \psi 1_{\rho \leq m} \leq 0
$$

Changing $\psi$ in $-\psi$, we conclude that $\int_{\Omega}(p-\varphi(\rho)) \psi 1_{\rho \leq m}=0$ for all $\psi \in C_{c}^{\infty}(\Omega, \mathbb{R})$. Once again by the Dominated Convergence Theorem, as $m$ to $+\infty$ we get: $\int_{\Omega}(p-$ $\varphi(\rho)) \psi=0$ for all $\psi \in C_{c}^{\infty}(\Omega)$ This gives $p=\varphi(\rho)$ a.e. in $\Omega$.

The hypothesis of convexity of the function $\varphi$ is only used to get that the four terms of the Right Hand Side of (3.17) are in $L^{1}(\Omega)$. If the hypothesis of convexity for $\varphi$ is replaced by the hypothesis (1.3), the proof is a little simpler. In this case, the $L^{2}$-bound of $p_{n}$ gives a $L^{2 \gamma}$-bound on $\rho_{n}$ (since $a \rho_{n}^{\gamma} \leq \varphi\left(\rho_{n}\right)+b=p_{n}+b$ ). Then one has $\rho_{n} \rightarrow \rho$ weakly in $L^{2 \gamma}(\Omega)$ and we can use $G_{n}$ with $\bar{\rho} \in L^{2 \gamma}(\Omega)$ such that $\varphi(\bar{\rho}) \in L^{2 \gamma /(2 \gamma-1)}(\Omega)$ (which is the dual space to $L^{2 \gamma}(\Omega)$ ). With such a $\bar{\rho}$, the four terms in the Right Hand Side of (3.17) are in $L^{1}(\Omega)$ ) and we obtain (3.18). For $\psi \in C_{c}^{\infty}(\Omega)$ and $k>0$, we take $\bar{\rho}=\rho+(1 / k) \psi$ (so that $\bar{\rho} \in L^{2 \gamma}(\Omega)$ and $\left.\varphi(\bar{\rho}) \in L^{2 \gamma /(2 \gamma-1)}(\Omega)\right)$. Passing to the limit as $k \rightarrow+\infty$ in (3.18) leads to

$$
\int_{\Omega}(p-\varphi(\rho)) \psi \mathrm{d} \boldsymbol{x} \leq 0 .
$$

With this inequality, we conclude, as before, that $p=\varphi(\rho)$ a.e. in $\Omega$.

In both cases ( $\varphi$ convex or $\varphi$ satisfies (1.3)), if $\varphi$ is increasing, we can obtain a strong convergence of $\rho_{n}$ and $p_{n}$, as in [6]. We take directly $\bar{\rho}=\rho$ in the definition of $G_{n}$. We obtain that $G_{n}=\left(\varphi\left(\rho_{n}\right)-\varphi(\rho)\right)\left(\rho_{n}-\rho\right) \rightarrow 0$ in $L^{1}(\Omega)$ as $n \rightarrow \infty$. Then, up to a subsequence, one has $G_{n} \rightarrow 0$ a.e. in $\Omega$. Since $\varphi$ is increasing, we finally deduce that $\rho_{n} \rightarrow \rho$ a.e.. This yields also $\rho_{n} \rightarrow \rho$ in $L^{q}(\Omega)$ for all $q \in[1,2[$, $p=\varphi(\rho)$ a.e. in $\Omega$ and $p_{n} \rightarrow p$ in $L^{q}(\Omega)$ for all $q \in[1,2[$.

The proof of Theorem 3.4 is now complete.

\section{Conclusion}

We gave a scheme for the discretization of the compressible Stokes problem with a general EOS and we proved the existence of a solution of the scheme along with the convergence of the approximate solution to an exact solution (up to a subsequence) as the mesh size goes to zero. A first difficulty of the paper is to get some estimates on the approximate solution (in particular with the dependancy of the forcing term with the density). A second complication is in the passage to the limit in the EOS. This difficulty is due to the nonlinearity of the EOS and the fact that the estimates on pressure and density only lead to weak convergences. It will be now interesting to consider the Navier-Stokes problem along with the evolution problem.

\section{Appendix A. Estimate on $p$}

Lemma A.1. Let $\Omega$ be a bounded set of $\mathbb{R}^{d}(d \geq 1)$ and $p \in L^{2}(\Omega), p \geq 0$ a.e.. We assume that there exist $a<1$ and $b \in \mathbb{R}$ such that

$$
\|p-m\|_{L^{2}} \leq a\|p\|_{L^{2}}+b,
$$

where $m$ is the mean value of $p$. Furthermore, we assume that there exist $A \in$ $\mathbb{R}$ and a continuous function $\psi$ from $\mathbb{R}_{+}$to $\mathbb{R}_{+}$such that $\int_{\Omega} \psi(p) d x \leq A$ and $\lim _{s \rightarrow \infty} \psi(s)=+\infty$. Then, there exists $C$ only depending on $\Omega, a, b$ A and $\psi$ such that $\|p\|_{L^{2}} \leq C$. 


\section{Proof of Lemma A.1}

We first modify the function $\psi$. Let $s_{0} \in \mathbb{R}_{+}$such that $\psi\left(s_{0}\right)>0$. We define $\bar{\psi}$ by

$$
\begin{aligned}
& \bar{\psi}(s)=\psi\left(s_{0}\right) \text { if } 0 \leq s \leq s_{0}, \\
& \bar{\psi}(s)=s \min _{t \in\left[s_{0}, s\right]} \frac{\psi(t)}{t} \text { if } s_{0}<s .
\end{aligned}
$$

We remark that $\bar{\psi}(s) \leq \psi(s)$ for $s \geq s_{0}$, so that $\int_{\Omega} \bar{\psi}(p) d x \leq \bar{A}=A+\psi\left(s_{0}\right) \lambda_{d}(\Omega)$. Furthermore, one has $\lim _{s \rightarrow+\infty} \bar{\psi}(s)=+\infty$. In order to prove this result, let $\left(s_{n}\right)_{n \in \mathbb{N}}$ be an incresing sequence such that $\lim _{n \rightarrow \infty} s_{n}=+\infty$. For $n \in \mathbb{N}$ let $t_{n} \in\left[s_{0}, s_{n}\right]$ such that $\bar{\psi}\left(s_{n}\right)=\left(\psi\left(t_{n}\right) / t_{n}\right) s_{n}$. For any converging (in $\mathbb{R}_{+} \cup\{+\infty\}$ ) subsequence of the sequence $\left(t_{n}\right)_{n \in \mathbb{N}}$, still denoted $\left(t_{n}\right)_{n \in \mathbb{N}}$, we have two possible cases,

First case. $\lim _{n \rightarrow \infty} t_{n}=t \in \mathbb{R}_{+}$. Then $\lim _{n \rightarrow \infty} \bar{\psi}\left(s_{n}\right)=+\infty$

(since $\psi(t) / t>0$ )

Second case. $\lim _{n \rightarrow \infty} t_{n}=+\infty$. Then $\lim _{n \rightarrow \infty} \bar{\psi}\left(s_{n}\right)=+\infty$

since $\bar{\psi}\left(s_{n}\right) \geq \psi\left(t_{n}\right)$.

We then conclude that $\lim _{s \rightarrow+\infty} \bar{\psi}(s)=+\infty$. Finally we also remark that the function $s \mapsto \frac{\bar{\psi}(s)}{s}$ is nonincreasing on $\mathbb{R}_{+}$.

We now prove the bound on $\|p\|_{L^{2}}$. Let $N>0$, one has

$$
\int_{\Omega} p(x) d x=\int_{p \geq N} p(x) d x+\int_{p<N} p(x) d x \leq \frac{1}{N} \int_{\Omega} p^{2}(x) d x+\frac{N}{\bar{\psi}(N)} \int_{\Omega} \bar{\psi}(p(x)) d x .
$$

This gives $m \lambda_{d}(\Omega) \leq \frac{1}{N}\|p\|_{L^{2}}{ }^{2}+\frac{N}{\bar{\psi}(N)} \bar{A}$. We now use the bound on $\|p-m\|_{L^{2}}$, it leads to

$$
\begin{aligned}
& \|p\|_{L^{2}} \leq\|p-m\|_{L^{2}}+m \lambda_{d}(\Omega)^{1 / 2} \\
& \leq a\|p\|_{L^{2}}+b+\frac{1}{N \lambda_{d}(\Omega)^{1 / 2}}\|p\|_{L^{2}}^{2}+\frac{N}{\bar{\psi}(N) \lambda_{d}(\Omega)^{1 / 2}} \bar{A} .
\end{aligned}
$$

If $\|p\|_{L^{2}} \neq 0$, we now choose $N$ such that $\frac{1}{N \lambda_{d}(\Omega)^{1 / 2}}=\frac{1-a}{2\|p\|_{L^{2}}}$, that is $N=$ $\frac{2\|p\|_{L^{2}}}{(1-a) \lambda_{d}(\Omega)^{1 / 2}}$, we obtain

$$
\frac{1-a}{2}\|p\|_{L^{2}} \leq b+\frac{2 \bar{A}}{\bar{\psi}(N)(1-a) \lambda_{d}(\Omega)}\|p\|_{L^{2}} .
$$

Since $\lim _{s \rightarrow \infty} \bar{\psi}(s)=+\infty$, there exists $C_{1}$ such that

$$
N \geq C_{1} \Rightarrow \frac{2 \bar{A}}{\bar{\psi}(N)(1-a) \lambda_{d}(\Omega)} \leq \frac{1-a}{4} .
$$

Then, with $C_{2}$ such that $\frac{2 C_{2}}{(1-a) \lambda_{d}(\Omega)^{1 / 2}}=C_{1}$, ona has

$$
\|p\|_{L^{2}} \geq C_{2} \Rightarrow \frac{2 \bar{A}}{\bar{\psi}(N)(1-a) \lambda_{d}(\Omega)} \leq \frac{1-a}{4} .
$$

Therefore

$$
\|p\|_{L^{2}} \geq C_{2} \Rightarrow\|p\|_{L^{2}} \leq \frac{4 b}{1-a} .
$$

Then, we conclude that $\|p\|_{L^{2}} \leq C=\max \left\{C_{2}, \frac{4 b}{1-a}\right\}$. 


\section{Appendix B. Passing to the limit in the EOS}

Lemma B.1. Let $\Omega$ be a bounded open set of $\mathbb{R}^{d}$. Let $\rho \in L^{2}(\Omega), \rho \geq 0$ a.e. in $\Omega$ and $u \in H_{0}^{1}(\Omega)^{d}$. Assume that $(\rho, u)$ satisfies:

$$
\int_{\Omega} \rho \boldsymbol{u} \cdot \nabla \varphi d x=0 \text { for all } \varphi \in W^{1, \infty}(\Omega) .
$$

Then,

$$
\int_{\Omega} \rho \operatorname{div}(\boldsymbol{u}) \mathrm{d} \boldsymbol{x}=0
$$

Remark B.2. Before giving the proof of Lemma B.1, we want to point out the following remark. In the case of a regular function $\rho$, say $\rho \in C^{1}(\bar{\Omega})$, and assuming that $\rho>0$ in $\Omega$, the proof is very easy. We take $\varphi=\ln (\rho)$ in (B.1) which yields, since $\nabla \varphi=\frac{1}{\rho} \nabla \rho$

$$
\int_{\Omega} u \cdot \nabla \rho \mathrm{d} \boldsymbol{x}=0 .
$$

But, for any $v \in C_{c}^{\infty}(\Omega)^{d}$ one has $\int_{\Omega} v \cdot \nabla \rho d x=-\int_{\Omega} \rho \operatorname{div}(v) \mathrm{d} \boldsymbol{x}$. Then, the density of $C_{c}^{\infty}(\Omega)^{d}$ in $H_{0}^{1}(\Omega)^{d}$ yields $\int_{\Omega} v \cdot \nabla \rho d x=-\int_{\Omega} \rho \operatorname{div}(v) \mathrm{d} \boldsymbol{x}$ for $v \in H_{0}^{1}(\Omega)^{d}$. This gives (B.2).

This proof is interesting because it suggests the proof of an equivalent result in the case of a discrete version (using a convenient numerical scheme) of $\operatorname{div}(\rho u)=0$ (see Lemma 3.1). In other words, working on a numerical scheme is quite similar of working on the continuous equation with a regular solution.

Proof. We now prove Lemma B.1. (without assuming $\rho \in C^{1}(\bar{\Omega})$ and $\rho>0$ ).

We set $u=0$ in $\mathbb{R}^{d} \backslash \Omega$ and $\rho=0$ in $\mathbb{R}^{d} \backslash \Omega$, we have $\rho \in L^{2}\left(\mathbb{R}^{d}\right)$ and $u \in H^{1}\left(\mathbb{R}^{d}\right)^{d}$. We also deduce from (B.1):

$$
\int_{\mathbb{R}^{d}} \rho u \cdot \nabla \varphi d x=0 \text { for all } \varphi \in C^{1}\left(\mathbb{R}^{d}\right) .
$$

Let $\left(r_{n}\right)_{n \in \mathbb{N}^{\star}}$ be a sequence of mollifiers, that is:

$$
\begin{aligned}
r \in C_{c}^{\infty}\left(\mathbb{R}^{d}, \mathbb{R}\right), \int_{\mathbb{R}^{d}} r d x=1, r & \geq 0 \text { in } \mathbb{R}^{d} \\
& \text { and, for } n \in \mathbb{N}^{\star}, x \in \mathbb{R}^{d}, r_{n}(x)=n^{d} r(n x) .
\end{aligned}
$$

For $n \in \mathbb{N}^{\star}$, we set $\rho_{n}=\rho \star r_{n}$ and $(\rho u)_{n}=(\rho u) \star r_{n}$. Thanks to (B.3), we have $\operatorname{div}\left((\rho u)_{n}\right)=0$ in $\mathbb{R}^{d}$. Since $u \in H^{1}\left(\mathbb{R}^{d}\right)^{d}$ and $\rho \in L^{2}\left(\mathbb{R}^{d}\right)$, we will prove in Lemma B.4 that $\operatorname{div}\left((\rho u)_{n}-\rho_{n} u\right) \rightarrow 0$ in $L^{1}\left(\mathbb{R}^{d}\right)$ as $n \rightarrow \infty$. Then, if $\left(q_{n}\right)_{n \in \mathbb{N}^{\star}}$ is a bounded sequence in $L^{\infty}\left(\mathbb{R}^{d}\right)$ which converges a.e. to $q$, we have:

$$
-\int_{\mathbb{R}^{d}} \operatorname{div}\left(\rho_{n} u\right) q_{n} d x=\int_{\mathbb{R}^{d}} \operatorname{div}\left((\rho u)_{n}-\rho_{n} u\right) q_{n} d x \rightarrow 0 \text { as } n \rightarrow \infty .
$$

Let $\psi$ be a bounded and $C^{1}$ function from $\mathbb{R}$ to $\mathbb{R}$, taking $q_{n}=\psi\left(\rho_{n}\right)$ in (B.5) (which converges a.e. to $\psi(\rho)$, at least up to a subsequence) we obtain

$$
-\int_{\mathbb{R}^{d}} \operatorname{div}\left(\rho_{n} u\right) \psi\left(\rho_{n}\right) d x \rightarrow 0 \text { as } n \rightarrow \infty .
$$


We now define $\theta$ by $\theta(s)=\int_{0}^{s} t \psi^{\prime}(t) d t$ for $s \in \mathbb{R}$ and we obtain

$$
\begin{array}{r}
\int_{\mathbb{R}^{d}} \theta\left(\rho_{n}\right) \operatorname{div}(u) d x=\int_{\mathbb{R}^{d}} \rho_{n} \psi^{\prime}\left(\rho_{n}\right) u \cdot \nabla \rho_{n} d x=\int_{\mathbb{R}^{d}} \rho_{n} u \cdot \nabla \psi\left(\rho_{n}\right) d x \\
=-\int_{\mathbb{R}^{d}} \operatorname{div}\left(\rho_{n} u\right) \psi\left(\rho_{n}\right) \mathrm{d} \boldsymbol{x},
\end{array}
$$

and then $\int_{\mathbb{R}^{d}} \theta(\rho) \operatorname{div}(u) d x=\lim _{n \rightarrow \infty} \int_{\mathbb{R}^{d}} \theta\left(\rho_{n}\right) \operatorname{div}(u) \mathrm{d} \boldsymbol{x}=0$.

It is now quite easy to construct a sequence of functions $\left(\psi_{n}\right)_{n \in \mathbb{N}}$ such that $0 \leq$ $\theta_{n}(s) \leq s$ for all $s \in \mathbb{R}_{+}$and $\lim _{n \rightarrow \infty} \theta_{n}(s)=s$ for all $s \in \mathbb{R}_{+}$. With the Dominated Convergence Theorem we then conclude that $\int_{\mathbb{R}^{d}} \rho \operatorname{div}(u) \mathrm{d} \boldsymbol{x}=0$.

Remark B.3. Under the hypothesis of Lemma B.1, a quick look on the proof of this lemma shows that it is also possible to prove

$$
\int_{\Omega} \psi(\rho) \operatorname{div}(\boldsymbol{u}) \mathrm{d} \boldsymbol{x}=0
$$

for any continuous function $\psi$ (from $\mathbb{R}$ to $\mathbb{R}$ ) "at most linear", that is such that

$$
\limsup _{s \rightarrow+\infty} \frac{|\psi(s)|}{s}<+\infty
$$

It is also possible (as it was said in Remark 1.3) to prove that $(\rho, u)$ is a renormalized solution to $\operatorname{div}(\rho u)=0$ in $\mathbb{R}^{d}$.

Indeed, let $\psi$ be a bounded and $C^{1}$ function from $\mathbb{R}$ to $\mathbb{R}$ and $\varphi \in C_{c}^{\infty}(\Omega)$. Taking $q_{n}=\psi\left(\rho_{n}\right) \varphi$ in (B.5) (which converges a.e. to $\psi(\rho) \varphi$, at least up to a subsequence) we obtain

$$
-\int_{\mathbb{R}^{d}} \operatorname{div}\left(\rho_{n} u\right) \psi\left(\rho_{n}\right) \varphi \mathrm{d} \boldsymbol{x} \rightarrow 0 \text { as } n \rightarrow \infty
$$

Taking, for $s \in \mathbb{R}, \bar{\psi}(s)=\int_{0}^{s} \psi(t) d t$ and $\theta(s)=\int_{0}^{s} t \psi^{\prime}(t) d t=s \psi(s)-\bar{\psi}(s)$, we obtain, after some integrations by parts and passing to the limit as $n \rightarrow \infty$,

$$
\int_{\mathbb{R}^{d}}\left(\rho \bar{\psi}^{\prime}(\rho)-\bar{\psi}(\rho)\right)(\operatorname{div} u) \varphi \mathrm{d} \boldsymbol{x}-\int_{\mathbb{R}^{d}} \bar{\psi}(\rho) u \cdot \nabla \varphi \mathrm{d} \boldsymbol{x}=0 .
$$

Then, it is easy to see that this equality also holds if $\bar{\psi}$ is a $C^{1}$ fonction form $\mathbb{R}$ to $\mathbb{R}$ with a bounded derivative. This proves that $(\rho, u)$ is a renormalized solution to $\operatorname{div}(\rho u)=0$ in $\mathbb{R}^{d}$.

Lemma B.4. Let $\rho \in L^{2}\left(\mathbb{R}^{d}\right)$ and $u \in H^{1}\left(\mathbb{R}^{d}\right)^{d}$. Let $\left(r_{n}\right)_{n \in \mathbb{N}^{\star}}$ be a sequence of mollifiers as given by (B.4) and, for $n \in \mathbb{N}^{\star}, \rho_{n}=\rho \star r_{n}$ and $(\rho u)_{n}=(\rho u) \star r_{n}$. Then,

and then,

$$
\boldsymbol{\nabla}\left((\rho u)_{n}-\rho_{n} u\right) \rightarrow 0 \text { in } L^{1}\left(\mathbb{R}^{d}\right)^{d \times d},
$$

$$
\operatorname{div}\left((\rho u)_{n}-\rho_{n} u\right) \rightarrow 0 \text { in } L^{1}\left(\mathbb{R}^{d}\right)
$$

Proof. Let $i, j \in\{1, \ldots, d\}$. Denoting by $u_{1}, \ldots, u_{d}$ the components of $u$ and by $\partial_{i}$ the derivative with respect to $x_{i}$, we have to prove that the sequence $\left(\partial_{i}\left[\left(\rho u_{j}\right)_{n}-\right.\right.$ $\left.\left.\rho_{n} u_{j}\right]\right)_{n \in \mathbb{N}^{\star}}$ converges to 0 in $L^{1}\left(\mathbb{R}^{d}\right)$. (As a consequence, taking $i=j$ and summing on $i$, we obtain that $\operatorname{div}\left((\rho u)_{n}-\rho_{n} u\right) \rightarrow 0$ in $L^{1}\left(\mathbb{R}^{d}\right)$.)

We have

$$
\partial_{i}\left[\left(\rho u_{j}\right)_{n}-\rho_{n} u_{j}\right]=\left(\rho u_{j}\right) \star \partial_{i} r_{n}-\left(\rho \star \partial_{i} r_{n}\right) u_{j}-\rho_{n} \partial_{i} u_{j}=F_{n}-G_{n},
$$


with

$$
F_{n}=\left(\rho u_{j}\right) \star \partial_{i} r_{n}-\left(\rho \star \partial_{i} r_{n}\right) u_{j}-\rho\left(\partial_{i} u_{j} \star r_{n}\right)
$$

and

$$
G_{n}=\rho_{n} \partial_{i} u_{j}-\rho\left(\partial_{i} u_{j} \star r_{n}\right) .
$$

Since $\rho_{n} \rightarrow \rho$ in $L^{2}\left(\mathbb{R}^{d}\right)$ and $\partial_{i} u_{j} \star r_{n} \rightarrow \partial_{i} u_{j}$ in $L^{2}\left(\mathbb{R}^{d}\right)($ as $n \rightarrow \infty)$, the two parts of $G_{n}$ converges in $L^{1}\left(\mathbb{R}^{d}\right)$ (as $\left.n \rightarrow \infty\right)$ to $\rho \partial_{i} u_{j}$. Then, the sequence $\left(G_{n}\right)_{n}$ converges in $L^{1}\left(\mathbb{R}^{d}\right)($ as $n \rightarrow \infty)$ to 0 . It remains to show that $F_{n} \rightarrow 0$ in $L^{1}\left(\mathbb{R}^{d}\right)$.

Using the fact that $\partial_{i} u_{j} \star r_{n}=u_{j} \star \partial_{i} r_{n}$ and the fact that $r_{n}$ has a compact support, we have, for a.e. $x \in \mathbb{R}^{d}$,

$$
\begin{aligned}
F_{n}(x) & =\int_{\mathbb{R}^{d}}(\rho(x-y)-\rho(x))\left(u_{j}(x-y)-u_{j}(x)\right) \partial_{i} r_{n}(y) d y \\
& =\int_{B}\left(\rho\left(x-\frac{z}{n}\right)-\rho(x)\right)\left(u_{j}\left(x-\frac{z}{n}\right)-u_{j}(x)\right) n \partial_{i} r(z) d z,
\end{aligned}
$$

where $B$ is a ball of center 0 and radius $R$ containing the support of $r$. Then, we get:

$$
\left|F_{n}(x)\right| \leq n \int_{B}\left|\left(\rho\left(x-\frac{z}{n}\right)-\rho(x)\right)\left(u_{j}\left(x-\frac{z}{n}\right)-u_{j}(x)\right)\right|\left|\partial_{i} r(z)\right| d z .
$$

We integrate over $\mathbb{R}$ the preceding inequality and we use the Fubini-Tonelli Theorem,

$$
\begin{aligned}
& \int_{\mathbb{R}^{d}}\left|F_{n}(x)\right| d x \leq \\
& n \int_{B}\left[\int_{\mathbb{R}^{d}}\left|\left(\rho\left(x-\frac{z}{n}\right)-\rho(x)\right)\left(u_{j}\left(x-\frac{z}{n}\right)-u_{j}(x)\right)\right| d x\right]\left|\partial_{i} r(z)\right| d z .
\end{aligned}
$$

Using the Cauchy-Schwarz Inequality, we have for $z \in B$,

$$
\begin{aligned}
& \int_{\mathbb{R}^{d}}\left|\left(\rho\left(x-\frac{z}{n}\right)-\rho(x)\right)\left(u_{j}\left(x-\frac{z}{n}\right)-u_{j}(x)\right)\right| d x \\
& \leq\left[\int_{\mathbb{R}^{d}}\left|\rho\left(x-\frac{z}{n}\right)-\rho(x)\right|^{2} d x\right]^{1 / 2}\left[\int_{\mathbb{R}^{d}}\left|u_{j}\left(x-\frac{z}{n}\right)-u_{j}(x)\right|^{2} d x\right]^{1 / 2} .
\end{aligned}
$$

For all $z \in B$ (see Lemma B.5) we have

$$
\int_{\mathbb{R}^{d}}\left|u_{j}\left(x-\frac{z}{n}\right)-u_{j}(x)\right|^{2} d x \leq\left(\frac{R}{n}\right)^{2}\|u\|_{H^{1}\left(\mathbb{R}^{d}\right)^{d}}^{2}
$$

Then,

$$
\begin{aligned}
& \int_{\mathbb{R}^{d}}\left|\left(\rho\left(x-\frac{z}{n}\right)-\rho(x)\right)\left(u_{j}\left(x-\frac{z}{n}\right)-u_{j}(x)\right)\right| d x \\
& \leq \frac{R}{n}\|u\|_{H^{1}\left(\mathbb{R}^{d}\right)^{d}}\left[\int_{\mathbb{R}^{d}}\left|\rho\left(x-\frac{z}{n}\right)-\rho(x)\right|^{2} d x\right]^{1 / 2} .
\end{aligned}
$$

Let $\varepsilon>0$. Since $\rho \in L^{2}\left(\mathbb{R}^{d}\right)$, there exists $\delta>0$ such that

$$
h \in \mathbb{R}^{d},|h| \leq \delta \Rightarrow\|\rho(\cdot+h)-\rho\|_{L^{2}\left(\mathbb{R}^{d}\right)} \leq \varepsilon .
$$

With (B.7), this gives if $n \geq R / \delta$ and $z \in B$,

$$
\int_{\mathbb{R}^{d}}\left|\left(\rho\left(x-\frac{z}{n}\right)-\rho(x)\right)\left(u_{j}\left(x-\frac{z}{n}\right)-u_{j}(x)\right)\right| d x \leq \varepsilon \frac{R}{n}\|u\|_{H^{1}\left(\mathbb{R}^{d}\right)^{d}} .
$$


Coming back to (B.6), we obtain, if $n \geq R / \delta$,

$$
\int_{\mathbb{R}^{d}}\left|F_{n}(x)\right| d x \leq n \frac{R}{n} \varepsilon\|u\|_{H^{1}\left(\mathbb{R}^{d}\right)^{d}} \int_{B}\left|\partial_{i} r(z)\right| d z=\varepsilon R\|u\|_{H^{1}\left(\mathbb{R}^{d}\right)^{d}} \int_{B}\left|\partial_{i} r(z)\right| d z .
$$

This proves that $F_{n} \rightarrow 0$ in $L^{1}\left(\mathbb{R}^{d}\right)$ as $n \rightarrow \infty$ and concludes the proof of Lemma B.4.

Lemma B.5. Let $w \in H^{1}\left(\mathbb{R}^{d}\right)$. Then, for $h \in \mathbb{R}^{d}$,

$$
\|w(\cdot+h)-w\|_{L^{2}\left(\mathbb{R}^{d}\right)} \leq|h|\|w\|_{H^{1}\left(\mathbb{R}^{d}\right)}
$$

where $|h|$ is the Euclidean norm of $h$.

Lemma B.5 is well-known. A proof is given, for instance, in [6].

The following lemma (Lemma B.6) proves that (for regular enough set $\Omega$ ) in Lemma B.1, $W^{1, \infty}(\Omega)$ can be replaced by $C_{c}^{\infty}(\Omega)$. That is to say that B.1 is true with $\varphi \in W^{1, \infty}(\Omega)$ if (and only if) it is true with the weaker assumption $\varphi \in C_{c}^{\infty}(\Omega)$. Lemma B.6 is given with $\rho \in L^{2}(\Omega)$ and $u \in\left(H_{0}^{1}(\Omega)\right)^{d}$, which is the case needed for the present paper (and allows a nice proof using the Hardy inequality). Similar results are possible with different assumptions on $u$ and $\rho$ (for instance, $\rho \in L^{\infty}(\Omega)$ and $\left.u \in W_{0}^{1,1}(\Omega)\right)$. However, the fact that $\rho u \in L^{1}(\Omega)$ is obviously not sufficient to ensure that (B.1) is true with $\varphi \in W^{1, \infty}(\Omega)$ as long as it is true for $\varphi \in C_{c}^{\infty}(\Omega)$. In a following paper, dealing with the Navier-Stokes equations, we will give the same lemma with a weaker assumption on $\rho$ (since $\rho \notin L^{2}(\Omega)$ in the case of the Navier-Stokes equations, when $d=3$ and $\gamma<\frac{5}{3}$ ). In this case, the proof will use a different argument, slightly more complicated.

Lemma B.6. Let $\Omega$ be a bounded open set of $\mathbb{R}^{d}$, with a Lipschitz continuous boundary. Let $u \in\left(H_{0}^{1}(\Omega)\right)^{d}$ and $\rho \in L^{2}(\Omega)$ such that, for all $\varphi \in C_{c}^{\infty}(\Omega)$,

$$
\int_{\Omega} \rho u \cdot \nabla \varphi d x=0 .
$$

Then (B.9) holds for all $\varphi \in W^{1, \infty}(\Omega)$.

The proof of this lemma is given in [7] (Lemma A.1).

Remark B.7. The hypothesis $\rho \in L^{2}(\Omega)$ is sharp in Lemma B.6, as we will see now. Let $d>1$ and $2 d /(d+2)<q<2$. We give here an example for which (B.9) holds for all $\varphi \in C_{c}^{\infty}(\Omega)$ but does not hold for some $\varphi \in W^{1, \infty}(\Omega)$. In this example, one has $\rho \in L^{q}(\Omega)$ and $u \in\left(H_{0}^{1}(\Omega)\right)^{d}$ (so that $\rho u \in L^{1}(\mathbb{R})^{d}$ ). Let us assume that $\Omega=] 0,2[\times]-1,1\left[^{d-1}\right.$. Let $\left.\alpha \in\right] \frac{1}{2}, \frac{1}{q}\left[\right.$. We define $\rho$ and $u=\left(u_{1}, \ldots, u_{d}\right)^{t}$ as follows:

$$
\begin{gathered}
u_{1}(x)=x_{1}^{\alpha} \prod_{i=2}^{d}\left(1-\left|x_{i}\right|\right) \text { if } x \in \Omega, x_{1} \leq 1, \\
u_{1}(x)=\left(2-x_{1}\right)^{\alpha} \prod_{i=2}^{d}\left(1-\left|x_{i}\right|\right) \text { if } x \in \Omega, x_{1}>1, \\
u_{2}=\ldots=u_{d}=0, \\
\rho(x)=\frac{1}{x_{1}^{\alpha}} \text { if } x \in \Omega, x_{1} \leq 1, \\
\rho(x)=\frac{1}{\left(2-x_{1}\right)^{\alpha}} \text { if } x \in \Omega, x_{1}>1 .
\end{gathered}
$$


We have $\rho \in L^{q}(\Omega)$ (thanks to $\alpha q<1$ ) and $u \in\left(H_{0}^{1}(\Omega)\right)^{d}$ (thanks to $2 \alpha>1$ ). Since $\rho u_{1}$ does not depend on $x_{1}$, if is easy to see (integrating by parts) that (B.9) holds for all $\varphi \in C_{c}^{\infty}(\Omega)$. Taking now $\varphi \in C_{c}^{\infty}\left(\mathbb{R}^{d}\right)$ with, for instance $\varphi=0$ outside ]$-1,1[\times]-\frac{1}{2}, \frac{1}{2}\left[{ }^{d-1}\right.$, one has

$$
\int_{\Omega} \rho u \cdot \nabla \varphi d x=-\int_{]-\frac{1}{2}, \frac{1}{2}[d-1} \prod_{i=2}^{d}\left(1-\left|x_{i}\right|\right) \varphi(0, y) d y,
$$

where $y=\left(x_{2}, \ldots, x_{n}\right)$. It is possible to choose $\varphi$ such that $\varphi(0, y)>0$ for all $y \in]-\frac{1}{2}, \frac{1}{2}\left[{ }^{d-1}\right.$. This gives $\int_{0} \rho u \cdot \nabla \varphi d x<0$ and proves that (B.1) does not hold for this choice of $\varphi$ (which belongs to $W^{1, \infty}(\Omega)$ ).

Lemma B.8. Let $\varphi$ be a convex function from $\mathbb{R}_{+}$to $\mathbb{R}_{+}$and $\left(\rho_{n}\right)_{n \in \mathbb{N}}$ be a sequence of nonnegative functions of $L^{2}(\Omega)$ weakly converging in $L^{2}(\Omega)$ to $\rho$. We assume that the sequence $\left(\varphi\left(\rho_{n}\right)\right)_{n \in \mathbb{N}}$ is bounded in $L^{2}(\Omega)$. Then, $\varphi(\rho) \in L^{2}(\Omega)$.

Proof. Since $\rho_{n} \geq 0$ a.e. (for all $n \in \mathbb{N}$ ), one has also $\rho \geq 0$ a.e..

Since the sequence $\left(\rho_{n}\right)_{n \in \mathbb{N}}$ weakly converge in $L^{2}(\Omega)$ to $\rho$, there exists a sequence $\left(\tilde{\rho}_{n}\right)_{n \in \mathbb{N}}$ converging (strongly) in $L^{2}(\Omega)$ to $\rho$ and such that $\tilde{\rho}_{n}$ is (for all $n \in \mathbb{N}$ ) a convex combination of $\left\{\rho_{k}, k \geq n\right\}$ (this result is known as the Mazur lemma). Then, for all $n \in \mathbb{N}$, there exists $q_{n} \in \mathbb{N}$ and $\alpha_{n, 0}, \ldots, \alpha_{n, q_{n}}$ such that

$$
\tilde{\rho}_{n}=\sum_{i=0}^{q_{n}} \alpha_{n, i} \rho_{n+i}, \sum_{i=0}^{q_{n}} \alpha_{n, i}=1 \text { and } \alpha_{n, i} \geq 0 \text { for } i=0, \ldots, q_{n} .
$$

Let $M=\sup \left\{\left\|\varphi\left(\rho_{n}\right)\right\|_{L^{2}(\Omega)}\right\}$. Using the convexity of $\varphi$ (and the fact that $\varphi$ take its values in $\mathbb{R}_{+}$) we have, for all $n \in \mathbb{N}$,

$$
0 \leq \varphi\left(\tilde{\rho}_{n}\right) \leq \sum_{i=0}^{q_{n}} \alpha_{n, i} \varphi\left(\rho_{n+i}\right) \text { a.e. }
$$

and then

$$
\left\|\varphi\left(\tilde{\rho}_{n}\right)\right\|_{L^{2}(\Omega)} \leq \sum_{i=0}^{q_{n}} \alpha_{n, i}\left\|\varphi\left(\rho_{n+i}\right)\right\|_{L^{2}(\Omega)} \leq M .
$$

Up to a subsequence, one has $\tilde{\rho}_{n} \rightarrow \tilde{\rho}$ a.e. and then, using the continuity of the function $\varphi, \varphi^{2}\left(\tilde{\rho}_{n}\right) \rightarrow \varphi^{2}(\rho)$ a.e on $\Omega$. Then, using Fatou Lemma, we thus get $\varphi(\rho) \in L^{2}(\Omega)\left(\right.$ and $\left.\|\varphi(\rho)\|_{L^{2}(\Omega)} \leq M\right)$.

\section{Appendix C. General lemmas}

Lemma C.1. Let $\left(F_{n}\right)_{n \in \mathbb{N}} \subset \mathrm{L}^{1}(\Omega)$ be an equi-integrable sequence, and $F$ be a function of $\mathrm{L}^{1}(\Omega)$. We assume that:

$$
\lim _{n \rightarrow \infty} \int_{\Omega} F_{n} \varphi \mathrm{d} \boldsymbol{x}=\int_{\Omega} F \varphi \mathrm{d} \boldsymbol{x} \text { for all } \varphi \in \mathrm{C}_{c}^{\infty}(\Omega) .
$$

Then:

$$
\lim _{n \rightarrow \infty} \int_{\Omega} F_{n} \mathrm{~d} \boldsymbol{x}=\int_{\Omega} F \mathrm{~d} \boldsymbol{x}
$$

Lemma C.1 is well-known. A proof is given, for instance, in [6]. The following lemma is also well-known. A simple proof of this result is given in [1]. 
Lemma C.2. Let $q \in \mathrm{L}^{2}(\Omega)$ such that $\int_{\Omega} q \mathrm{~d} \boldsymbol{x}=0$. Then, there exists $\boldsymbol{w} \in$ $\left(\mathrm{H}_{0}^{1}(\Omega)\right)^{d}$ such that $\operatorname{div}(\boldsymbol{w})=q$ a.e. in $\Omega$ and $\|\boldsymbol{w}\|_{\mathrm{H}^{1}(\Omega)^{d}} \leq c_{2}\|q\|_{\mathrm{L}^{2}(\Omega)}$ where $c_{2}$ only depends on $\Omega$.

We now give two simple lemmas related to the so-called "M-matrices". We recall that for a vector $x$ of $\mathbb{R}^{n}$, the fact that all the components of $x$ are nonnegative is denoted by $x \geq 0$. Similarly the fact that all the components of $x$ are positive is denoted by $x>0$.

Lemma C.3. Let $n \in \mathbb{N}^{\star}$ and $A$ be a $n \times n$ matrix with real entries (these entries are denoted by $\left.a_{i, j}, i, j=1, \ldots, n\right)$. We asume that $A$ satisfies the following properties:

$$
\left\{\begin{array}{l}
a_{i, j} \leq 0 \text { for all } i, j \in\{1, \ldots, n\}, i \neq j \\
a_{i, i}+\sum_{j \neq i} a_{i, j}>0 \text { for all } i \in\{1, \ldots, n\} .
\end{array}\right.
$$

then,

$$
x \in \mathbb{R}^{n}, A x \geq 0 \Rightarrow x \geq 0,
$$

which is equivalent to say that $A$ is invertible and that all the entries of $A^{-1}$ are nonnegatives. Futhermore, one also has

$$
x \in \mathbb{R}^{n}, A x>0 \Rightarrow x>0,
$$

Proof. The proof of (C.2) is very classical. We can do it, for instance, by contradiction. Let $x \in \mathbb{R}^{n}$ such that $A x \geq 0$. We assume that $\alpha=\min \left\{x_{i}, i=1, \ldots, n\right\}$ $<0$ (where the $x_{i}$ are the components of $x$ ) and we choose $i_{0} \in\{1, \ldots, n\}$ such that $x_{i_{0}}=\alpha$.

Since the $i_{0}$-component of $A x$ is nonnegative and since $x_{i_{0}} \leq x_{i}$ for all $i$, one has, thanks to the properties of $A$,

$$
x_{i_{0}}\left(a_{i_{0}, i_{0}}+\sum_{j \neq i_{0}} a_{i_{0}, j}\right) \geq 0,
$$

Which gives $x_{i_{0}} \geq 0$, in contradiction with $x_{i_{0}}=\alpha<0$. This proves (C.2).

In order to prove (C.3). Let $e$ be the vector of $\mathbb{R}^{n}$ whose all components are equal to 1. let $x \in \mathbb{R}^{n}$ such $A x>0$. Then, for $\varepsilon>0$ small enough, one has $A(x-\varepsilon e)=A x-\varepsilon A e \geq 0$. Thanks to (C.2), one deduces $x-\varepsilon e \geq 0$ and this gives $x>0$.

The second lemma is a little bit less classical but is a very simple consequence of the first one.

Lemma C.4. Let $n \in \mathbb{N}^{\star}$ and $A$ be a $n \times n$ matrix with real entries (these entries are denoted by $\left.a_{i, j}, i, j=1, \ldots, n\right)$. We asume that $A$ satisfies the following properties:

$$
\left\{\begin{array}{l}
a_{i, j} \leq 0 \text { for all } i, j \in\{1, \ldots, n\}, i \neq j, \\
a_{i, i}+\sum_{j \neq i} a_{j, i}>0 \text { for all } i \in\{1, \ldots, n\} .
\end{array}\right.
$$

then,

$$
x \in \mathbb{R}^{n}, A x \geq 0 \Rightarrow x \geq 0
$$

and

$$
x \in \mathbb{R}^{n}, A x>0 \Rightarrow x>0,
$$


Proof. The matrix $A^{t}$ satisfies the properties of lemma C.3. Then $A^{t}$ is invertible and $\left(A^{t}\right)^{-1}$ has all its entries nonnegative. This gives that $A$ is also invertible and has all its entries nonnegative since $\left(A^{t}\right)^{-1}=\left(A^{-1}\right)^{t}$. This gives that $A$ satisfies (C.4)

The proof of (C.5) is the same as the proof of (C.3) in lemma C.3.

Lemma C.5. Let $\varphi$ be a function of class $C^{1}$ from $\mathbb{R}_{+}^{\star}$ to $\mathbb{R}$. Let $\psi$ from $\mathbb{R}_{+}^{\star}$ to $\mathbb{R}$ such that $s \psi^{\prime}(s)=\varphi^{\prime}(s)$ for all $s \in \mathbb{R}_{+}^{\star}$. Let $a, b \in \mathbb{R}_{+}^{*}, a \neq b$. Then, there exists $c$ between $a$ et $b$ such that

$$
(\psi(b)-\psi(a)) b-(\varphi(b)-\varphi(a))=\frac{1}{2}(b-a)^{2} \psi^{\prime}(c) .
$$

Proof. One has

$$
(\psi(b)-\psi(a)) b-(\varphi(b)-\varphi(a))=b \int_{a}^{b} \psi^{\prime}(s) d s-\int_{a}^{b} \varphi^{\prime}(s) d s=\int_{a}^{b}(b-s) \psi^{\prime}(s) d s .
$$

But,

$$
\min _{t \in[a, b]} \psi^{\prime}(t) \int_{a}^{b}(b-s) d s \leq \int_{a}^{b}(b-s) \psi^{\prime}(s) d s \leq \max _{t \in[a, b]} \psi^{\prime}(t) \int_{a}^{b}(b-s) d s .
$$

Then, since $\psi^{\prime}$ is continuous on $[a, b]$, there exists $c \in[a, b]$ such that

$$
\int_{a}^{b}(b-s) \psi^{\prime}(s) d s=\psi^{\prime}(c) \int_{a}^{b}(b-s) d s .
$$

Noticing that $\int_{a}^{b}(b-s) d s=\frac{1}{2}(b-a)^{2}$, we obtain the desired equality.

\section{REFERENCES}

[1] Bramble, J.H.. A proof of the inf-sup condition for the Stokes equations on Lipschitz domains, Mathematical Models and Methods in Applied Sciences, 13, 2003

[2] Ciarlet, P. G. Finite Elements Methods - Basic Error Estimates for Elliptic Problems. Handbook of Numerical Analysis, Volume II, North Holland, 1991, editor: Ciarlet, P. and Lions, J.L..

[3] M. Crouzeix and P.-A. Raviart. Conforming and nonconforming finite element methods for solving the stationary Stokes equations I. Revue Française d'Automatique, Informatique et Recherche Opérationnelle (R.A.I.R.O.), R-3:33-75, 1973.

[4] R. DiPerna and P.-L. Lions. Ordinary differential equations, transport theory and Sobolev spaces. Invent. Math. 98 (1989), no. 3, 511-547

[5] A. Ern and J.-L. Guermond. Theory and practice of finite elements. Number 159 in Applied Mathematical Sciences. Springer, New York, 2004.

[6] R. Eymard, T. Gallouët, R. Herbin, and J.-C. Latché. A convergent finite element-finite volume scheme for the compressible Stokes problem. Part II: the isentropic case. Math. Comp. 79 (2010), no. 270, 649675

[7] R. Eymard, T. Gallouët, R. Herbin, and J.-C. Latché. Convergence of the Mac scheme for the compressible Stokes equations. SIAM J. Numer. Anal. 48 (2010), no. 6, 22182246

[8] E. Feireisl. Dynamics of viscous compressible fluids. Oxford Lecture Series in Mathematics and its Applications, 26. Oxford University Press, Oxford, 2004

[9] A. Fettah and T. Gallouët. Compressible Stokes problem with general EOS. Finite volumes for complex applications. VI. Problems \& perspectives. Volume 1, 2, 457465, Springer Proc. Math., 4, Springer, Heidelberg, 2011.

[10] T. Gallouët, R. Herbin, and J.-C. Latché. A convergent finite element-finite volume scheme for the compressible Stokes problem. Part I: the isothermal case. Math. Comp. 78 (2009), no. 267,13331352 
[11] K. Karlsen, and T. Karper. A convergent nonconforming finite element method for compressible Stokes flow. SIAM J. Numer. Anal. 48 (2010), no. 5, 18461876

[12] P.-L. Lions. Mathematical topics in fluid mechanics -volume 2- compressible models. volume 10 of Oxford Lecture Series in Mathematics and its Applications. Oxford University Press, 1998.

[13] A. Novotnỳ, and I. Straškraba. Introduction to the mathematical theory of compressible flow. Oxford Lecture Series in Mathematics and its Applications, 27. Oxford University Press, Oxford, 2004 